\title{
Organizational Agility Assessment of a Moroccan Healthcare Organization in Times of COVID-19
}

Fadoua Tamtam*, Amina Tourabi

Systems Engineering and Decision Support Laboratory, National School of Applied Sciences, University IBN ZOHR, Agadir, 80000, Morocco

\author{
A R T I C L E I N F O \\ Article history: \\ Received: .05 June, 2020 \\ Accepted: 23 July, 2020 \\ Online: 19 August, 2020
}

Keywords:

COVID-19

Organization agility

Assessment model

\begin{abstract}
A B S T R A C T
Since its appearance, COVID-19 has severely impacted the healthcare sector all over the world. The healthcare organizations should be agile in order to cope with this new health crisis. Indeed, organization agility was highly recommended as an essential basis for flexibility, innovation, speed, as well competitiveness. Different research provided different conceptual models suitable to evaluate the organization agility. In this sense, this paper presents an assessment model, which by defining different agile enablers, criteria and attributes, aims at identifying the least and the most suitable enablers influencing the healthcare organization agility. To realize it practically, this paper uses the fuzzy logic approach which provides the improvement directions for enhancing the organization agility. Subsequently, the data gathered from a Moroccan healthcare organization was substituted in this assessment model and the level and the suggestions improvement for agility were derived. In this way, the organization will integrate the successful combination of the agility enablers in this dynamic environment.
\end{abstract}

\section{Introduction}

The story of the pandemic "COVID-19', began in 2019 when the first case were identified from Wuhan, China [1]. Since its first appearance, COVID-19 has been receiving an increasing attention by academic and executive specialists and many researches have been developed on it in order to provide a general definition of the virus. In the beginning, COVID-19 has created a global healthcare crisis, and then it disrupted other sectors: economic, environmental and social [2]. But perhaps the most significant pressure was for the healthcare organizations which strengthened their medical system [3] in order to enhance their responsiveness, adaptability, flexibility, which explains the importance of agility implementation in the healthcare sector through the outbreaks of COVID 19.

Agility concept was presented as the effective exploration of different competitive bases by including the suitable resources and practices in order to cope with the changing environment $[4,5]$. Later, different proposals of agility definitions have been derived and which presented a general consensus [5]: It means the organization capacity to react quickly [5]-[11] to the varied changes in market demand [8]-[11] in terms of cost, specification, quality, quantity and delivery $[11,12]$. Despite being defined in different ways and from different perspectives, agility has sometimes been used interchangeably to refer to concepts such as

${ }^{*}$ Corresponding Author: Fadoua Tamtam, fadoua.tamtam@gmail.com adaptability, flexibility, speed, intelligence or sharpness. In contrast to this point of view, several authors have expressed the difference between these concepts, which justifies our choice to use the word "Agility" many times in our paper.

In order to evaluate the agility of an organization [5], several approaches such as system approach $[5,13]$, graph theory $[5,14]$, multi-grade fuzzy logic [15], regression analysis [5, 16] and other artificial intelligence techniques, such as neural network [15], neuro-fuzzy [15], have been used [5, 13, 14, 16-19] . A main objective of this study is to help the healthcare organization to implement an easier and less complicated practical tool in order to evaluate their agility [11]. The above purpose suggests an assessment model in which we evaluated the enablers influencing the adoption of agility [15].

Our paper is organized as follows: In the next paragraph, we review previous researches related to agile enablers. By presenting the fuzzy logic approach, we presented the required steps to apply this methodology to a real case. Moreover, the results provided are discussed and the limits of the study and suggestions for future research are finally presented [11].

\section{Literature review: Agile enablers}

According to different conceptual models of agility presented in literature, companies can benefit from different enablers [11] in order to achieve agility. These enablers, also known as providers 


\section{F. Tamtam et al. / Advances in Science, Technology and Engineering Systems Journal Vol. 5, No. 4, 567-576 (2020)}

or levers [20], were introduced by Gunasekaran [21, 22] in order to identify the required features of the agile organization [20]. In his study, he identified seven agile enablers: virtual corporation formation tools/metrics, physically distributed teams and manufacturing, quick partnership formation, concurrent engineering, integrated information system, quick prototyping tools and E-commerce [22]. In 1999, Yusuf et al. [4] presented different enablers under ten groups: the introduction of new products, the formation of partnerships, continuous improvement, short conception/production of deadlines, decentralized decisionmaking, response to market requirements ...etc [20]. Later, Sharifi and his colleagues proposed four enablers from four different areas: organization, people, technology and innovation [9,23]. Based on their sample, Tolf et al. identified five essentials enablers for an agile organization: transparent and transient inter-organizational links at all levels, market sensitivity and customer focus, management by support for self-organizing employees, organic structures and flexible human and resource capacity for timely delivery [24]. In their paper [25], Lin and his colleagues suggested four agility enablers: collaborative relationships, process integration, information integration and customer sensitivity [26]. Other enablers were identified by Eshlaghy et al., as organizational structure, virtual organization, information technology, organizational culture, leadership, team working, empowerment and improvement, motivation system and planning and evaluation performance [27].
From this literature review, we can notice that there is no single list of agility enablers [20] which is due to the varied requirements of each organization [28]. However, all the enablers should have some criteria and attributes that make them agile. For example, the criterion called "Organizational structure" should be flexible to accept changes, this means that the different attributes of the organizational structure should be easily adaptable [20], while promoting a fluid flow of information [15], communication [29] and knowledge [30], which makes it possible to accept the interchangeability of employees [15] and focus on teamwork [20, 27, 30, 31] . For the other criterion "Processes", it should be flexible [20,30], promote and concentrate on external environment developments [20, 30, 32]. According to Sherehiy et al. [30], human resource agility, as an enabler of the agile organization [20], should be flexible [33], multi-skilled [15, 33], adaptable, resilient $[20,30,32]$, able to cooperate $[15,20,30]$, take personal initiative and cope well with changes $[20,30,32]$. The technology enabler should also be flexible like other enablers, modular and easily scalable [20].

Summarizing the above literature, different enablers, as listed in Table 1, are chosen as necessary conditions for organizational agility [33]. Table 1 suggest an assessment model in which we defined, firstly, the agile enablers that should be implemented by organizations; secondly, for each enabler different agile criteria are listed and finally agile attributes are identified in order to achieve the required agile criteria [15].

Table 1: Organizational agility enablers (Adapted from [12, 15, 27, 29-34])

\begin{tabular}{|c|c|c|}
\hline Agile enablers & Agile criteria & Agile attributes \\
\hline \multirow{16}{*}{$\begin{array}{c}\text { Management } \\
\text { responsibility agility } \\
\left(\mathrm{E}_{1}\right)\end{array}$} & \multirow{4}{*}{$\begin{array}{l}\text { Organizational } \\
\text { structure } \\
\left(E_{11}\right)\end{array}$} & $\begin{array}{l}\text { Flattened, horizontal organizational structure that promotes innovation, training and having an open } \\
\text { information, communication and knowledge policy }\left(\mathrm{E}_{111}\right)\end{array}$ \\
\hline & & Fluid information flow $\left(\mathrm{E}_{112}\right)$ \\
\hline & & Staff interchangeability $\left(\mathrm{E}_{113}\right)$ \\
\hline & & Collaborative and team work $\left(\mathrm{E}_{114}\right)$ \\
\hline & \multirow{5}{*}{$\begin{array}{l}\text { Devolution of } \\
\text { authority } \\
\left(E_{12}\right)\end{array}$} & Clear definition of staff responsibility and authority $\left(\mathrm{E}_{121}\right)$ \\
\hline & & Training to create self-managed and multi-functional teams $\left(\mathrm{E}_{122}\right)$ \\
\hline & & Decentralized decision-making, knowledge and control $\left(\mathrm{E}_{123}\right)$ \\
\hline & & Loyalty and commitment to a project or a group $\left(\mathrm{E}_{124}\right)$ \\
\hline & & Authority change when tasks change $\left(\mathrm{E}_{125}\right)$ \\
\hline & \multirow{7}{*}{$\begin{array}{l}\text { Nature of management } \\
\qquad\left(\mathrm{E}_{13}\right)\end{array}$} & Participative management style $\left(\mathrm{E}_{131}\right)$ \\
\hline & & Clearly known management purpose $\left(\mathrm{E}_{132}\right)$ \\
\hline & & Management participation and support $\left(\mathrm{E}_{133}\right)$ \\
\hline & & Motivation of profit associated with a humanitarian approach $\left(\mathrm{E}_{134}\right)$ \\
\hline & & Regular conduct of employer-employees meetings $\left(\mathrm{E}_{135}\right)$ \\
\hline & & Quick evaluation and implementation of employee suggestions $\left(\mathrm{E}_{136}\right)$ \\
\hline & & Less strict or few rules and procedures $\left(\mathrm{E}_{137}\right)$ \\
\hline \multirow{7}{*}{$\begin{array}{c}\text { Manufacturing } \\
\text { management agility } \\
\left(\mathrm{E}_{2}\right)\end{array}$} & \multirow{5}{*}{$\begin{array}{l}\text { Patient response adoption } \\
\qquad\left(\mathrm{E}_{21}\right)\end{array}$} & Dominance of the culture of continuous improvement $\left(\mathrm{E}_{211}\right)$ \\
\hline & & Communication media to collect responses $\left(\mathrm{E}_{212}\right)$ \\
\hline & & Incorporating patient feedback into services $\left(\mathrm{E}_{213}\right)$ \\
\hline & & Staff empowerment to resolve patient issues $\left(\mathrm{E}_{214}\right)$ \\
\hline & & Efficient information system and technology $\left(\mathrm{E}_{215}\right)$ \\
\hline & \multirow{2}{*}{$\begin{array}{l}\text { Change in business and } \\
\text { technical processes }\end{array}$} & Flexible business system $\left(\mathrm{E}_{221}\right)$ \\
\hline & & Application of business process reengineering to reinvent and reorganize the organization $\left(\mathrm{E}_{222}\right)$ \\
\hline
\end{tabular}


F. Tamtam et al. / Advances in Science, Technology and Engineering Systems Journal Vol. 5, No. 4, 567-576 (2020)

\begin{tabular}{|c|c|c|}
\hline & $\left(\mathrm{E}_{22}\right)$ & Positive employee attitude towards change, new ideas and technology $\left(\mathrm{E}_{223}\right)$ \\
\hline & & Risk management $\left(\mathrm{E}_{224}\right)$ \\
\hline & & Adopting supply chain management concepts to improve the efficiency of outsourcing $\left(\mathrm{E}_{231}\right)$ \\
\hline & Outsourcing & Exploitation of information technology (IT) in supply chain management $\left(\mathrm{E}_{232}\right)$ \\
\hline & $\left(\mathrm{E}_{23}\right)$ & Involvement of suppliers and different agents in product/service development $\left(\mathrm{E}_{233}\right)$ \\
\hline & & Working with fewer qualified suppliers $\left(\mathrm{E}_{234}\right)$ \\
\hline & $\begin{array}{c}\text { Processes sensing } \\
\left(E_{24}\right)\end{array}$ & Promoting and concentrating on external environment developments $\left(\mathrm{E}_{241}\right)$ \\
\hline & & Reconfigurable process $\left(\mathrm{E}_{251}\right)$ \\
\hline & Processes responding & Scalable process $\left(\mathrm{E}_{252}\right)$ \\
\hline & & Simple process to implement $\left(\mathrm{E}_{253}\right)$ \\
\hline & & Process design $\left(\mathrm{E}_{261}\right)$ \\
\hline & Concurrent engineering & Intelligent Engineering Design Support System $\left(\mathrm{E}_{262}\right)$ \\
\hline & $\left(\mathrm{E}_{26}\right)$ & Integrated multidisciplinary teams of customers and suppliers $\left(\mathrm{E}_{263}\right)$ \\
\hline & & Continuous reengineering of the organization and business processes based on benchmarking $\left(\mathrm{E}_{264}\right)$ \\
\hline & & Flexible employees to accept the adoption of new technologies $\left(E_{311}\right)$ \\
\hline & Employee st & Multi-skilled and flexible staff $\left(\mathrm{E}_{312}\right)$ \\
\hline & $\left(\mathrm{E}_{31}\right)$ & Implementation of job rotation system $\left(\mathrm{E}_{313}\right)$ \\
\hline & & Education and training for all the existing and new employees $\left(\mathrm{E}_{314}\right)$ \\
\hline & Employee involvement & Employee cooperation $\left(\mathrm{E}_{321}\right)$ \\
\hline & $\left(\mathrm{E}_{32}\right)$ & Employee empowerment $\left(\mathrm{E}_{322}\right)$ \\
\hline & & Entrepreneurial organizational culture $\left(\mathrm{E}_{331}\right)$ \\
\hline & & Reward programs to encourage innovation and based on financial and non-financial measures $\left(\mathrm{E}_{332}\right)$ \\
\hline & management practices & Multi-skill training improving organizational agility $\left(\mathrm{E}_{333}\right)$ \\
\hline & & Multi-functional, developed and trained employees $\left(\mathrm{E}_{334}\right)$ \\
\hline Human resource agility & & Development of differentiation and diversity $\left(\mathrm{E}_{335}\right)$ \\
\hline & & Anticipation of problems linked to change and resolution of these problems $\left(\mathrm{E}_{341}\right)$ \\
\hline & Human resources capacities & Personal initiative $\left(\mathrm{E}_{342}\right)$ \\
\hline & $\left(\mathrm{E}_{34}\right)$ & Interpersonal and cultural adaptability $\left(\mathrm{E}_{343}\right)$ \\
\hline & & Resiliency $\left(\mathrm{E}_{344}\right)$ \\
\hline & & Personal, informal, goal-oriented and spontaneous coordination $\left(\mathrm{E}_{351}\right)$ \\
\hline & Coordination & Network communication $\left(\mathrm{E}_{352}\right)$ \\
\hline & & Management-employee cohesion $\left(\mathrm{E}_{353}\right)$ \\
\hline & Human knowledre and & Knowledge and skills management systems $\left(\mathrm{E}_{361}\right)$ \\
\hline & skills & Protection of sensitive information $\left(\mathrm{E}_{362}\right)$ \\
\hline & & Knowledge acquisition from internal and external sources $\left(\mathrm{E}_{363}\right)$ \\
\hline & & Flexible manufacturing setups $\left(\mathrm{E}_{411}\right)$ \\
\hline & & Less time to change machine settings $\left(\mathrm{E}_{412}\right)$ \\
\hline & Manufacturing set-ups & Modernization of machines $\left(\mathrm{E}_{413}\right)$ \\
\hline & $\left(\mathrm{E}_{41}\right)$ & Usage of collapsible set-ups, Jigs and Fixtures $\left(\mathrm{E}_{414}\right)$ \\
\hline & & Usage of automated tools $\left(\mathrm{E}_{415}\right)$ \\
\hline Technology agility & & Active policy to keep work areas clean and tidy $\left(\mathrm{E}_{416}\right)$ \\
\hline$\left(\mathrm{E}_{4}\right)$ & & Specification of product life to the patient $\left(\mathrm{E}_{421}\right)$ \\
\hline & Product life cycle & Company encourages patient to switch to new product $\left(\mathrm{E}_{422}\right)$ \\
\hline & $\left(\mathrm{E}_{42}\right)$ & $\begin{array}{l}\text { Products superior field performance for a stipulated period with least } \\
\text { maintenance cost }\left(\mathrm{E}_{423}\right)\end{array}$ \\
\hline & Product service & Products designed for easy serviceability $\left(\mathrm{E}_{431}\right)$ \\
\hline & $\left(\mathrm{E}_{43}\right)$ & Products incorporated with a modular design $\left(\mathrm{E}_{432}\right)$ \\
\hline
\end{tabular}


F. Tamtam et al. / Advances in Science, Technology and Engineering Systems Journal Vol. 5, No. 4, 567-576 (2020)

\begin{tabular}{|c|c|c|}
\hline & & Service centers well equipped with spares $\left(\mathrm{E}_{433}\right)$ \\
\hline & & $\begin{array}{l}\text { Minimum time required to execute the planning and to restore the defective product to its original } \\
\text { performance }\left(\mathrm{E}_{434}\right)\end{array}$ \\
\hline & \multirow{4}{*}{$\begin{array}{l}\text { Production methodology } \\
\qquad\left(\mathrm{E}_{44}\right)\end{array}$} & Management's interest towards investment on flexible manufacturing system (FMS) concepts $\left(\mathrm{E}_{441}\right)$ \\
\hline & & Application of Lean manufacturing principles for waste elimination $\left(\mathrm{E}_{442}\right)$ \\
\hline & & Development of products whose components are all outsourced and assembled in-house $\left(\mathrm{E}_{443}\right)$ \\
\hline & & IT application for better supplier management $\left(\mathrm{E}_{444}\right)$ \\
\hline & \multirow{5}{*}{$\begin{array}{l}\text { Manufacturing planning } \\
\qquad\left(\mathrm{E}_{45}\right)\end{array}$} & Execution of short range planning $\left(\mathrm{E}_{451}\right)$ \\
\hline & & Organization's procurement policy based on time schedule $\left(\mathrm{E}_{452}\right)$ \\
\hline & & Strategic network in supply chain management to exercise zero inventory system $\left(\mathrm{E}_{453}\right)$ \\
\hline & & Improved manufacturing technology $\left(\mathrm{E}_{454}\right)$ \\
\hline & & Structured and flexible manufacturing processes $\left(\mathrm{E}_{455}\right)$ \\
\hline & \multirow{2}{*}{$\begin{array}{l}\text { IT integration } \\
\left(\mathrm{E}_{46}\right)\end{array}$} & IT utilities incorporated with reengineered pattern of working $\left(\mathrm{E}_{461}\right)$ \\
\hline & & Electronic commerce [27] $\left(\mathrm{E}_{462}\right)$ \\
\hline & \multirow{4}{*}{$\begin{array}{l}\text { Customization } \\
\qquad\left(\mathrm{E}_{47}\right)\end{array}$} & Rapid introduction of new products/services $\left(\mathrm{E}_{471}\right)$ \\
\hline & & Responding to changing market requirements $\left(\mathrm{E}_{472}\right)$ \\
\hline & & Products with high added value $\left(\mathrm{E}_{473}\right)$ \\
\hline & & First-time correct design $\left(\mathrm{E}_{474}\right)$ \\
\hline \multirow{12}{*}{$\begin{array}{c}\text { Manufacturing } \\
\text { strategy agility } \\
\left(\mathrm{E}_{5}\right)\end{array}$} & \multirow{3}{*}{$\begin{array}{l}\text { Status of quality } \\
\qquad\left(E_{51}\right)\end{array}$} & Products/services exceeding patient expectations $\left(\mathrm{E}_{511}\right)$ \\
\hline & & Carrying out surveys/studies to guarantee the quality status $\left(\mathrm{E}_{512}\right)$ \\
\hline & & Usage of total quality management tools $\left(\mathrm{E}_{513}\right)$ \\
\hline & & Improved productivity in all functions $\left(\mathrm{E}_{521}\right)$ \\
\hline & Status of productivity & Reduction of non value-adding costs $\left(\mathrm{E}_{522}\right)$ \\
\hline & & Quality is not infused at the cost of productivity $\left(\mathrm{E}_{523}\right)$ \\
\hline & & Costing and product pricing system focused on value-added and non-value-added activities $\left(\mathrm{E}_{531}\right)$ \\
\hline & $\begin{array}{l}\text { Cost management } \\
\qquad\left(\mathrm{E}_{53}\right)\end{array}$ & Costing system enabling the evaluation of future resource consumption $\left(\mathrm{E}_{532}\right)$ \\
\hline & & Product cost fixed according to the pricing of the customer $\left(\mathrm{E}_{533}\right)$ \\
\hline & & Scheduled activities $\left(\mathrm{E}_{541}\right)$ \\
\hline & $\begin{array}{l}\text { Time management } \\
\left(\mathrm{E}_{54}\right)\end{array}$ & IT based communication system $\left(\mathrm{E}_{542}\right)$ \\
\hline & & Adoption of time compression technologies $\left(\mathrm{E}_{543}\right)$ \\
\hline
\end{tabular}

\section{Fuzzy logic methodology to evaluate organizational agility}

In order to enhance organizational agility in practice, the use of different methods and tools were recommended in literature [11, 23]. Focusing on methodological articles [11], the fuzzy logic approach has been used to assess the current agility level and identify the weaker attributes that need a particular attention to enhance the organizational agility. This approach is preferred over other methodologies because it can take the linguistic data as input, then convert linguistic expressions into corresponding fuzzy intervals and finally express the results back in linguistic terms with the help of Fuzzy Agility Index (FAI) [5].

Many studies in literature have used fuzzy logic to measure agility level of the healthcare organization (e.g. [5, 35]). Taking cues from these papers, this study uses this approach to evaluate the agility of a Moroccan healthcare organization.

\section{Numerical illustration of fuzzy logic approach}

\subsection{About the healthcare organization}

Our study has been done at a public hospital (referred as HealthOrg), located in Morocco and where patients can carry out the diagnosis of COVID-19. In order to cope with the new dynamic environment, HealthOrg aims to strengthen its agility level. However, it found it difficult to identify enablers that influence its agility, in particular the weaker ones which need to be improved [15]. In this context, we aimed to evaluate the agility of HealthOrg.

Table 2 provides an illustration of different steps to apply the fuzzy logic approach [11].

Table 2: Steps required applying the fuzzy logic methodology (Adapted from [5, 32])

\begin{tabular}{|l|}
\hline \multicolumn{1}{|c|}{ Steps } \\
\hline $\begin{array}{l}\text { Identify a list of agile enablers that influence the organizational } \\
\text { agility. }\end{array}$ \\
\hline $\begin{array}{l}\text { Define the linguistic variables for evaluating performance } \\
\text { rating and importance weights of agile attributes. }\end{array}$ \\
\hline $\begin{array}{l}\text { Approximate the linguistic terms by the corresponding fuzzy } \\
\text { intervals. }\end{array}$ \\
\hline Calculate the FAI of the organization. \\
\hline Match the FAI with the appropriate linguistic level. \\
\hline Calculate Fuzzy Performance Importance Index (FPII). \\
\hline
\end{tabular}




\subsection{Fuzzy logic application}

- Step1: Identification of agile enablers, criteria and attributes [32]: By identifying a list of five agile enablers from the literature [5], twenty-six criteria and ninety-eight attributes were identified (Table 1).

- Step 2: Definition of the linguistic variables for evaluating performance rating and importance weights of agile attributes [32]: Following this list, five experts $(\mathrm{E} 1, \mathrm{E} 2, \ldots, \mathrm{E} 5)$ from HealthOrg were asked to provide the weights in terms of linguistic variables ranging from "Very low (VL)" to "Very High (VH)" and ratings in terms of linguistic variables ranging from "Worst (W)" to "Excellent (E)" [5] (Table 3).

Table 3: Importance weight and performance rating of agile attributes

\begin{tabular}{|c|c|c|c|c|c|c|c|c|c|c|}
\hline \multirow{2}{*}{ 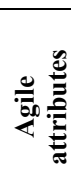 } & \multicolumn{5}{|c|}{ Importance weight } & \multicolumn{5}{|c|}{ Performance rating } \\
\hline & $\mathbf{E}_{1}$ & $\mathbf{E}_{2}$ & $E_{3}$ & $\mathbf{E}_{4}$ & $\mathbf{E}_{5}$ & $\mathbf{E}_{1}$ & $\mathbf{E}_{2}$ & $\mathbf{E}_{3}$ & $\mathbf{E}_{4}$ & $\mathbf{E}_{5}$ \\
\hline$E_{111}$ & $\mathrm{H}$ & $\mathrm{FH}$ & $\mathrm{M}$ & $\mathrm{H}$ & $\mathrm{FH}$ & $\mathrm{E}$ & VG & VG & $\mathrm{G}$ & $\mathrm{F}$ \\
\hline $\mathbf{E}_{112}$ & $\mathrm{H}$ & $\mathrm{M}$ & $\mathrm{FH}$ & $\mathrm{FH}$ & $\mathrm{H}$ & $\mathrm{P}$ & $\mathrm{P}$ & $\mathrm{F}$ & $\mathrm{G}$ & $\mathrm{G}$ \\
\hline $\mathbf{E}_{113}$ & $\mathrm{FH}$ & $\mathrm{H}$ & $\mathrm{FH}$ & $\mathrm{H}$ & $\mathrm{FH}$ & $\mathrm{VP}$ & $\mathrm{W}$ & $\mathrm{G}$ & $\mathrm{F}$ & $\mathrm{F}$ \\
\hline $\mathbf{E}_{114}$ & $\mathrm{H}$ & $\mathrm{M}$ & $\mathrm{H}$ & $\mathrm{FH}$ & $\mathrm{FH}$ & $\mathrm{E}$ & $\mathrm{G}$ & $\mathrm{G}$ & $\mathrm{F}$ & $\mathrm{F}$ \\
\hline $\mathbf{E}_{121}$ & $\mathrm{H}$ & $\mathrm{M}$ & $\mathrm{FH}$ & $\mathrm{M}$ & $\mathrm{FH}$ & $\mathrm{F}$ & $\mathrm{F}$ & $\mathrm{G}$ & $\mathrm{VG}$ & VG \\
\hline $\mathbf{E}_{122}$ & $\mathrm{H}$ & $\mathrm{FH}$ & $\mathrm{M}$ & $\mathrm{H}$ & FH & $\mathrm{P}$ & F & $\mathrm{P}$ & $\mathrm{G}$ & $\mathrm{F}$ \\
\hline $\mathbf{E}_{123}$ & $\mathrm{FH}$ & $\mathrm{H}$ & $\mathrm{FH}$ & $\mathrm{M}$ & $\mathrm{FH}$ & $\mathrm{W}$ & $\mathrm{W}$ & $\mathrm{P}$ & $\mathrm{F}$ & $\mathrm{P}$ \\
\hline $\mathbf{E}_{124}$ & $\mathrm{H}$ & $\mathrm{H}$ & $\mathrm{M}$ & $\mathrm{FH}$ & $\mathrm{H}$ & $\mathrm{W}$ & $\mathrm{W}$ & VP & $\mathrm{F}$ & $\mathrm{F}$ \\
\hline $\mathbf{E}_{125}$ & $\mathrm{M}$ & $\mathrm{H}$ & $\mathrm{H}$ & $\mathrm{FH}$ & $\mathrm{H}$ & $\mathrm{G}$ & $\mathrm{VG}$ & $\mathrm{E}$ & $\mathrm{E}$ & $\mathrm{G}$ \\
\hline $\mathbf{E}_{131}$ & $\mathrm{VH}$ & $\mathrm{H}$ & $\mathrm{H}$ & $\mathrm{VH}$ & $\mathrm{H}$ & $\mathrm{G}$ & $\mathrm{F}$ & $\mathrm{G}$ & $\mathrm{G}$ & $\mathrm{VG}$ \\
\hline $\mathbf{E}_{132}$ & $\mathrm{H}$ & $\mathrm{FH}$ & $\mathrm{H}$ & $\mathrm{FH}$ & $\mathrm{FH}$ & $\mathrm{E}$ & $\mathrm{E}$ & $\mathrm{G}$ & $\mathrm{F}$ & $\mathrm{E}$ \\
\hline $\mathbf{E}_{133}$ & $\mathrm{H}$ & $\mathrm{FH}$ & $\mathrm{H}$ & FH & $\mathrm{H}$ & $\mathrm{G}$ & $\mathrm{G}$ & $\mathrm{G}$ & $\mathrm{VG}$ & $\mathrm{G}$ \\
\hline $\mathbf{E}_{134}$ & $\mathrm{FH}$ & $\mathrm{M}$ & $\mathrm{FH}$ & $\mathrm{M}$ & $\mathrm{FH}$ & $\mathrm{F}$ & $\mathrm{G}$ & $\mathrm{F}$ & $\mathrm{F}$ & $\mathrm{G}$ \\
\hline $\mathbf{E}_{135}$ & $\mathrm{H}$ & $\mathrm{M}$ & $\mathrm{M}$ & $\mathrm{M}$ & $\mathrm{H}$ & $\mathrm{G}$ & $\mathrm{G}$ & $\mathrm{G}$ & VG & $\mathrm{F}$ \\
\hline $\mathbf{E}_{133}$ & $\mathrm{VH}$ & $\mathrm{H}$ & $\mathrm{H}$ & $\mathrm{VH}$ & $\mathrm{VH}$ & VP & $\mathrm{F}$ & $\mathrm{P}$ & $\mathrm{P}$ & $\mathrm{G}$ \\
\hline $\mathbf{E}_{137}$ & $\mathrm{H}$ & $\mathrm{FH}$ & $\mathrm{M}$ & $\mathrm{FH}$ & $\mathrm{H}$ & $\mathrm{W}$ & $\mathrm{F}$ & $\mathrm{G}$ & $\mathrm{F}$ & $\mathrm{G}$ \\
\hline $\mathbf{E}_{211}$ & $\mathrm{H}$ & $\mathrm{H}$ & $\mathrm{H}$ & $\mathrm{FH}$ & $\mathrm{H}$ & $\mathrm{G}$ & $\mathrm{VG}$ & $\mathrm{G}$ & $\mathrm{F}$ & $\mathrm{F}$ \\
\hline $\mathbf{E}_{212}$ & $\mathrm{FH}$ & $\mathrm{M}$ & $\mathrm{FH}$ & $\mathrm{FH}$ & $M$ & $\mathrm{~W}$ & $\mathrm{~W}$ & $\mathrm{~F}$ & $\mathrm{G}$ & VP \\
\hline $\mathbf{E}_{213}$ & $\mathrm{FH}$ & $\mathrm{FH}$ & $\mathrm{FH}$ & $\mathrm{FH}$ & $\mathrm{FH}$ & VP & $\mathrm{P}$ & $\mathrm{P}$ & $\mathrm{F}$ & $\mathrm{P}$ \\
\hline $\mathbf{E}_{214}$ & $\mathrm{VH}$ & $\mathrm{H}$ & $\mathrm{H}$ & $\mathrm{VH}$ & $\mathrm{H}$ & $\mathrm{F}$ & $\mathrm{G}$ & $\mathrm{E}$ & VG & $\mathrm{E}$ \\
\hline $\mathbf{E}_{215}$ & $\mathrm{H}$ & $\mathrm{FH}$ & $\mathrm{H}$ & $\mathrm{FH}$ & $\mathrm{FH}$ & VP & $\mathrm{P}$ & $\mathrm{W}$ & $\mathrm{F}$ & $\mathrm{F}$ \\
\hline $\mathbf{E}_{221}$ & FL & $\mathrm{M}$ & $\mathrm{FL}$ & FL & M & $\mathrm{VG}$ & $\mathrm{F}$ & $\mathrm{E}$ & $\mathrm{F}$ & $\mathrm{G}$ \\
\hline $\mathbf{E}_{222}$ & $\mathrm{H}$ & $\mathrm{FH}$ & $\mathrm{H}$ & $\mathrm{M}$ & FH & W & VP & $\mathrm{G}$ & $\mathrm{E}$ & $\mathrm{G}$ \\
\hline $\mathbf{E}_{223}$ & $\mathrm{H}$ & $\mathrm{M}$ & $\mathrm{M}$ & $\mathrm{FH}$ & $\mathrm{H}$ & VP & $\mathrm{F}$ & $\mathrm{F}$ & $\mathrm{W}$ & $\mathrm{E}$ \\
\hline $\mathbf{E}_{224}$ & FH & $\mathrm{H}$ & $\mathrm{H}$ & $\mathrm{H}$ & FH & $\mathrm{F}$ & $\mathrm{G}$ & $\mathrm{E}$ & VG & $\mathrm{G}$ \\
\hline $\mathbf{E}_{231}$ & $\mathrm{VH}$ & $\mathrm{H}$ & $\mathrm{VH}$ & $\mathrm{H}$ & $\mathrm{H}$ & $\mathrm{F}$ & $\mathrm{F}$ & $\mathrm{G}$ & $\mathrm{F}$ & VG \\
\hline $\mathbf{E}_{232}$ & $\mathrm{H}$ & $\mathrm{M}$ & $\mathrm{FH}$ & $\mathrm{H}$ & $\mathrm{FH}$ & VP & $\mathrm{P}$ & $\mathrm{G}$ & $\mathrm{F}$ & $\mathrm{W}$ \\
\hline $\mathbf{E}_{233}$ & $\mathrm{H}$ & $\mathrm{H}$ & $\mathrm{H}$ & $\mathrm{H}$ & $\mathrm{H}$ & $\mathrm{F}$ & $\mathrm{W}$ & VP & $\mathrm{F}$ & $\mathrm{G}$ \\
\hline $\mathbf{E}_{234}$ & FH & $\mathrm{H}$ & M & FH & FH & $\mathrm{E}$ & VG & $\mathrm{F}$ & $\mathrm{G}$ & $\mathrm{G}$ \\
\hline $\mathbf{E}_{241}$ & $\mathrm{H}$ & $\mathrm{M}$ & $\mathrm{FH}$ & $\mathrm{FH}$ & $\mathrm{FH}$ & VP & $\mathrm{F}$ & $\mathrm{G}$ & $\mathrm{G}$ & $\mathrm{F}$ \\
\hline $\mathbf{E}_{251}$ & $\mathrm{H}$ & $\mathrm{FH}$ & $\mathrm{M}$ & $\mathrm{H}$ & $\mathrm{M}$ & $\mathrm{F}$ & $\mathrm{VG}$ & $\mathrm{VG}$ & $\mathrm{G}$ & VG \\
\hline $\mathbf{E}_{252}$ & $\mathrm{FH}$ & $\mathrm{FH}$ & $\mathrm{FH}$ & FH & $\mathrm{FH}$ & VP & $\mathrm{VG}$ & $\mathrm{F}$ & $\mathrm{G}$ & $\mathrm{F}$ \\
\hline $\mathbf{E}_{253}$ & $\mathrm{FH}$ & $\mathrm{M}$ & $\mathrm{FH}$ & $\mathrm{FH}$ & $\mathrm{M}$ & W & $\mathrm{W}$ & VP & $\mathrm{F}$ & VP \\
\hline $\mathbf{E}_{261}$ & $\mathrm{H}$ & FH & $\mathrm{H}$ & $\mathrm{FH}$ & $\mathrm{M}$ & $\mathrm{G}$ & $\mathrm{F}$ & $\mathrm{G}$ & $\mathrm{VG}$ & $\mathrm{E}$ \\
\hline$E_{262}$ & $\mathrm{H}$ & $\mathrm{M}$ & $\mathrm{FH}$ & $\mathrm{H}$ & $\mathrm{H}$ & W & W & $\mathrm{G}$ & $F$ & $\mathrm{G}$ \\
\hline $\mathbf{E}_{263}$ & FH & $\mathrm{M}$ & $\mathrm{H}$ & FH & FH & VP & $\mathrm{P}$ & $\mathrm{W}$ & $P$ & $\mathrm{~F}$ \\
\hline $\mathbf{E}_{264}$ & $\mathrm{H}$ & $\mathrm{FH}$ & $\mathrm{H}$ & $\mathrm{FH}$ & $M$ & $\mathrm{G}$ & $F$ & $P$ & VP & $\mathrm{G}$ \\
\hline $\mathbf{E}_{311}$ & $\mathrm{FH}$ & $\mathrm{FH}$ & $\mathrm{H}$ & $\mathrm{FH}$ & $\mathrm{H}$ & $P$ & $\mathrm{~W}$ & $F$ & $\mathrm{G}$ & $\mathrm{P}$ \\
\hline $\mathbf{E}_{312}$ & $\mathrm{H}$ & $\mathrm{H}$ & $\mathrm{H}$ & $\mathrm{H}$ & $\mathrm{H}$ & $\mathrm{W}$ & $\mathrm{W}$ & $\mathrm{F}$ & $\mathrm{F}$ & $\mathrm{P}$ \\
\hline $\mathbf{E}_{313}$ & $\mathrm{VH}$ & $\mathrm{H}$ & $\mathrm{VH}$ & $\mathrm{VH}$ & $\mathrm{H}$ & $\mathrm{F}$ & $\mathrm{G}$ & $\mathrm{P}$ & $P$ & $\mathrm{P}$ \\
\hline $\mathbf{E}_{314}$ & FH & $\mathrm{H}$ & $\mathrm{FH}$ & $\mathrm{M}$ & $\mathrm{FH}$ & $\mathrm{E}$ & VG & $\mathrm{G}$ & $\mathrm{E}$ & $\mathrm{F}$ \\
\hline$E_{321}$ & $\mathrm{H}$ & $\mathrm{H}$ & $\mathrm{H}$ & $\mathrm{H}$ & $\mathrm{H}$ & $\mathrm{E}$ & $\mathrm{E}$ & VG & $F$ & $\mathrm{~F}$ \\
\hline $\mathbf{E}_{322}$ & FH & $\mathrm{M}$ & $\mathrm{H}$ & $\mathrm{H}$ & $\mathrm{H}$ & $\mathrm{P}$ & $\mathrm{F}$ & $\mathrm{F}$ & $\mathrm{G}$ & $\mathrm{VG}$ \\
\hline $\mathbf{E}_{331}$ & $\mathrm{H}$ & $\mathrm{FH}$ & $\mathrm{H}$ & $\mathrm{H}$ & $\mathrm{M}$ & $\mathrm{E}$ & VG & $\mathrm{G}$ & $F$ & $\mathrm{G}$ \\
\hline $\mathbf{E}_{332}$ & $\mathrm{H}$ & $\mathrm{H}$ & $\mathrm{H}$ & $\mathrm{H}$ & $\mathrm{H}$ & $\mathrm{E}$ & $\mathrm{F}$ & $\mathrm{G}$ & $\mathrm{F}$ & $\mathrm{F}$ \\
\hline $\mathbf{E}_{332}$ & $\mathrm{H}$ & $\mathrm{M}$ & $\mathrm{M}$ & $\mathrm{M}$ & $\mathrm{M}$ & $\mathrm{W}$ & $\mathrm{W}$ & $\mathrm{F}$ & $\mathrm{G}$ & $\mathrm{F}$ \\
\hline $\mathbf{E}_{334}$ & FH & FH & $\overline{\mathrm{H}}$ & $\mathrm{H}$ & FH & $\mathrm{W}$ & $P$ & VP & $\mathrm{G}$ & $\mathrm{F}$ \\
\hline $\mathbf{E}_{335}$ & $\mathrm{H}$ & $\mathrm{FH}$ & $\mathrm{FH}$ & $\mathrm{H}$ & $\mathrm{H}$ & $\mathrm{E}$ & $\mathrm{E}$ & $\mathrm{E}$ & $\mathrm{G}$ & $\mathrm{E}$ \\
\hline
\end{tabular}

\begin{tabular}{|c|c|c|c|c|c|c|c|c|c|c|}
\hline $\mathbf{E}_{341}$ & $\mathrm{H}$ & $\mathrm{M}$ & $\mathrm{FH}$ & $\mathrm{M}$ & $\mathrm{H}$ & VP & $\mathrm{P}$ & $\mathrm{F}$ & $\mathrm{G}$ & $\mathrm{F}$ \\
\hline $\mathbf{E}_{342}$ & $\mathrm{H}$ & $\mathrm{FH}$ & FH & $\mathrm{FH}$ & $\mathrm{M}$ & $\bar{F}$ & $\mathrm{G}$ & $P$ & $F$ & $\mathrm{G}$ \\
\hline $\mathbf{E}_{343}$ & $\mathrm{H}$ & $\mathrm{FH}$ & $\mathrm{H}$ & $\mathrm{FH}$ & $\mathrm{M}$ & $F$ & $E$ & VG & $\mathrm{G}$ & $\bar{F}$ \\
\hline $\mathbf{E}_{344}$ & $\mathrm{H}$ & $\mathrm{FH}$ & $\mathrm{FH}$ & $\mathrm{H}$ & $\mathrm{H}$ & $\mathrm{G}$ & $\mathrm{F}$ & $\mathrm{F}$ & $\mathrm{E}$ & $\mathrm{G}$ \\
\hline$E_{351}$ & $\mathrm{H}$ & $\mathrm{H}$ & $\mathrm{FH}$ & $\mathrm{H}$ & $\mathrm{M}$ & $\mathrm{VP}$ & $\mathrm{F}$ & $\mathrm{F}$ & $F$ & $F$ \\
\hline $\mathbf{E}_{352}$ & FH & $\mathrm{M}$ & $\mathrm{M}$ & FH & FH & $E$ & $\mathrm{G}$ & $\mathrm{G}$ & $\mathrm{F}$ & $\mathrm{F}$ \\
\hline $\mathbf{E}_{353}$ & $\mathrm{H}$ & $\mathrm{FH}$ & $\mathrm{H}$ & $\mathrm{FH}$ & $\mathrm{FH}$ & $\mathrm{G}$ & $\mathrm{E}$ & $\mathrm{F}$ & $\mathrm{F}$ & VG \\
\hline $\mathbf{E}_{361}$ & $\mathrm{M}$ & $\mathrm{M}$ & $\mathrm{M}$ & $\mathrm{FH}$ & FH & W & W & $\mathrm{W}$ & $\mathrm{F}$ & $\mathrm{P}$ \\
\hline $\mathbf{E}_{362}$ & $\mathrm{H}$ & $\mathrm{M}$ & $\mathrm{FH}$ & $\mathrm{FH}$ & $\mathrm{H}$ & $\mathrm{E}$ & $\mathrm{E}$ & $\mathrm{E}$ & $E$ & VG \\
\hline $\mathbf{E}_{363}$ & $\mathrm{H}$ & $\mathrm{FH}$ & $\mathrm{H}$ & $\mathrm{H}$ & $\mathrm{H}$ & $E$ & $\mathrm{VG}$ & $\mathrm{F}$ & $\mathrm{P}$ & $\bar{F}$ \\
\hline $\mathbf{E}_{411}$ & $\mathrm{M}$ & $\mathrm{H}$ & $\mathrm{M}$ & $\mathrm{M}$ & $\mathrm{FH}$ & $\mathrm{VG}$ & $\mathrm{G}$ & $\mathrm{F}$ & $\mathrm{E}$ & $E$ \\
\hline $\mathbf{E}_{412}$ & FH & $\mathrm{FH}$ & $\mathrm{M}$ & $\mathrm{M}$ & $\mathrm{H}$ & W & $\mathrm{G}$ & $P$ & $\mathrm{~F}$ & $\mathrm{~F}$ \\
\hline $\mathbf{E}_{413}$ & $\mathrm{H}$ & M & $\mathrm{M}$ & M & $\mathrm{FH}$ & $\mathrm{G}$ & $\mathrm{F}$ & $\mathrm{G}$ & $\mathrm{E}$ & $\mathrm{F}$ \\
\hline $\mathbf{E}_{414}$ & FH & $\mathrm{H}$ & $\mathrm{H}$ & $\mathrm{H}$ & $\mathrm{H}$ & $\mathrm{G}$ & $\mathrm{F}$ & $\mathrm{F}$ & $\mathrm{F}$ & $\mathrm{VG}$ \\
\hline $\mathbf{E}_{415}$ & $\mathrm{M}$ & $\mathrm{H}$ & FH & $\mathrm{H}$ & $\mathrm{H}$ & $\mathrm{E}$ & $\mathrm{G}$ & $F$ & $P$ & VP \\
\hline $\mathbf{E}_{416}$ & $\mathrm{FH}$ & $\mathrm{H}$ & $\mathrm{M}$ & $\begin{array}{l}\mathrm{H} \\
\end{array}$ & $\mathrm{FH}$ & $\mathrm{W}$ & $\mathrm{VP}$ & $\mathrm{P}$ & VP & $\mathrm{F}$ \\
\hline $\mathbf{E}_{421}$ & $\mathrm{H}$ & $\mathrm{VH}$ & $\mathrm{H}$ & $\mathrm{H}$ & $\mathrm{H}$ & $E$ & $\mathrm{~F}$ & $\mathrm{G}$ & $\mathrm{E}$ & $\mathrm{VG}$ \\
\hline $\mathbf{E}_{422}$ & FH & $\mathrm{FH}$ & $\mathrm{FH}$ & $\mathrm{M}$ & $\mathrm{H}$ & $\mathrm{E}$ & $\mathrm{E}$ & $\mathrm{G}$ & $\mathrm{G}$ & $\mathrm{G}$ \\
\hline $\mathbf{E}_{423}$ & $\mathrm{H}$ & $\mathrm{FH}$ & $\mathrm{M}$ & M & $\mathrm{FH}$ & $\mathrm{E}$ & $\mathrm{VG}$ & $\mathrm{F}$ & $\mathrm{G}$ & $\mathrm{G}$ \\
\hline $\mathbf{E}_{431}$ & $\mathrm{H}$ & $\mathrm{H}$ & $\mathrm{FH}$ & $\mathrm{H}$ & $\mathrm{FH}$ & $\mathrm{G}$ & $\mathrm{G}$ & $\mathrm{VG}$ & $\mathrm{E}$ & $\mathrm{E}$ \\
\hline $\mathbf{E}_{432}$ & FL & FL & $\mathrm{L}$ & $\mathrm{VL}$ & $\mathrm{M}$ & $\mathrm{E}$ & $\mathrm{VG}$ & $\mathrm{G}$ & VG & $\mathrm{E}$ \\
\hline $\mathbf{E}_{433}$ & FL & $\mathrm{M}$ & $\mathrm{FH}$ & $\mathrm{H}$ & $\mathrm{FH}$ & $\mathrm{G}$ & $\mathrm{F}$ & $\mathrm{E}$ & $\mathrm{E}$ & VG \\
\hline $\mathbf{E}_{434}$ & $\mathrm{M}$ & $\mathrm{FH}$ & $\mathrm{FH}$ & $\mathrm{H}$ & $\mathrm{VH}$ & $\mathrm{VP}$ & $P$ & $\mathrm{~F}$ & $\mathrm{VG}$ & $\mathrm{VG}$ \\
\hline $\mathbf{E}_{441}$ & FH & $\mathrm{VH}$ & $\mathrm{M}$ & $\mathrm{FH}$ & $\mathrm{VH}$ & $E$ & $\mathrm{VG}$ & $\mathrm{VG}$ & VG & $\mathrm{G}$ \\
\hline $\mathbf{E}_{442}$ & $\mathrm{H}$ & $\mathrm{M}$ & $\mathrm{H}$ & $\mathrm{FH}$ & $\mathrm{H}$ & $\mathrm{G}$ & $\mathrm{F}$ & VG & $\mathrm{E}$ & VP \\
\hline $\mathbf{E}_{443}$ & FL & $\mathrm{H}$ & $\mathrm{VH}$ & $\mathrm{H}$ & $\mathrm{FH}$ & $\mathrm{G}$ & $\mathrm{F}$ & VP & $\mathrm{G}$ & $\mathrm{F}$ \\
\hline $\mathbf{E}_{444}$ & $\mathrm{VH}$ & $\mathrm{H}$ & $\mathrm{H}$ & FH & $\mathrm{VH}$ & $\mathrm{G}$ & $\mathrm{E}$ & $\mathrm{F}$ & VG & $\mathrm{F}$ \\
\hline$E_{451}$ & $\mathrm{VH}$ & $\mathrm{VH}$ & $\mathrm{H}$ & $\mathrm{H}$ & $\mathrm{FH}$ & W & $\mathrm{VP}$ & $\mathrm{F}$ & G & $F$ \\
\hline $\mathbf{E}_{452}$ & $\mathrm{VH}$ & $\mathrm{VH}$ & $\mathrm{H}$ & $\mathrm{VH}$ & $\mathrm{H}$ & $\mathrm{W}$ & $\mathrm{W}$ & $\mathrm{G}$ & $\mathrm{F}$ & $\bar{F}$ \\
\hline $\mathbf{E}_{453}$ & FH & $\mathrm{VH}$ & $\mathrm{FH}$ & $\mathrm{VH}$ & $\mathrm{M}$ & VP & $\mathrm{P}$ & $\mathrm{G}$ & $\mathrm{VG}$ & $\mathrm{F}$ \\
\hline $\mathbf{E}_{454}$ & $\mathrm{VH}$ & $\mathrm{VH}$ & $\mathrm{H}$ & $\mathrm{FH}$ & $\mathrm{FH}$ & $\mathrm{G}$ & $\mathrm{F}$ & $\mathrm{F}$ & VP & $\mathrm{P}$ \\
\hline $\mathbf{E}_{455}$ & $\mathrm{M}$ & $\mathrm{H}$ & $\mathrm{VH}$ & $\mathrm{H}$ & $\mathrm{H}$ & $E$ & $\mathrm{VG}$ & $\mathrm{G}$ & $\mathrm{VG}$ & $\mathrm{F}$ \\
\hline $\mathbf{E}_{461}$ & FH & FL & $\mathrm{FL}$ & FH & FH & $\mathrm{G}$ & $\mathrm{G}$ & $\mathrm{F}$ & $\mathrm{F}$ & $\mathrm{F}$ \\
\hline $\mathbf{E}_{462}$ & FH & $\mathrm{M}$ & $\mathrm{FH}$ & $\mathrm{FH}$ & $\mathrm{H}$ & VG & $\mathrm{E}$ & $\mathrm{E}$ & $\mathrm{F}$ & $\mathrm{G}$ \\
\hline $\mathbf{E}_{471}$ & $\mathrm{H}$ & $\mathrm{H}$ & $\mathrm{FH}$ & $\mathrm{H}$ & $\mathrm{H}$ & $F$ & $\mathrm{~F}$ & G & VG & VG \\
\hline$E_{472}$ & FL & $\mathrm{FH}$ & $\mathrm{FH}$ & $\mathrm{L}$ & $\mathrm{FH}$ & $E$ & $\mathrm{E}$ & $\mathrm{E}$ & $\mathrm{VG}$ & $\mathrm{G}$ \\
\hline $\mathbf{E}_{473}$ & $\mathrm{H}$ & $\mathrm{H}$ & $\mathrm{FH}$ & FH & $\mathrm{H}$ & $\mathrm{G}$ & $\begin{array}{l}\mathrm{E} \\
\end{array}$ & VG & $\mathrm{F}$ & $\mathrm{F}$ \\
\hline $\mathbf{E}_{474}$ & $\mathrm{VH}$ & $\mathrm{M}$ & $\mathrm{VH}$ & $\mathrm{H}$ & $\mathrm{VH}$ & W & $\mathrm{VP}$ & $\mathrm{F}$ & $\mathrm{G}$ & $\mathrm{E}$ \\
\hline $\mathbf{E}_{511}$ & FH & FL & $\mathrm{FL}$ & $\mathrm{M}$ & $\mathrm{FH}$ & $\mathrm{F}$ & $\mathrm{F}$ & $\mathrm{G}$ & $\mathrm{F}$ & $\mathrm{F}$ \\
\hline $\mathbf{E}_{512}$ & FH & $\mathrm{FH}$ & $\mathrm{FH}$ & $\mathrm{FH}$ & $\mathrm{H}$ & $E$ & $\mathrm{VP}$ & $\mathrm{E}$ & $\mathrm{F}$ & $\mathrm{G}$ \\
\hline $\mathbf{E}_{513}$ & $\mathrm{H}$ & $\mathrm{H}$ & $\mathrm{M}$ & $\mathrm{H}$ & $\mathrm{H}$ & $E$ & $\mathrm{E}$ & $\mathrm{E}$ & $\mathrm{E}$ & $\mathrm{E}$ \\
\hline $\mathbf{E}_{521}$ & $\mathrm{H}$ & $\mathrm{FH}$ & $\mathrm{VH}$ & $\mathrm{H}$ & $\mathrm{H}$ & VP & $\mathrm{G}$ & $\mathrm{E}$ & $\mathrm{G}$ & $\mathrm{G}$ \\
\hline $\mathbf{E}_{522}$ & $\mathrm{VH}$ & $\mathrm{M}$ & $\mathrm{VH}$ & $\mathrm{H}$ & $\mathrm{VH}$ & VP & $P$ & $\mathrm{P}$ & W & $\mathrm{G}$ \\
\hline $\mathbf{E}_{523}$ & $\mathrm{H}$ & $\mathrm{VH}$ & $\mathrm{FH}$ & $\mathrm{FL}$ & FL & $\mathrm{E}$ & $\mathrm{VG}$ & $\mathrm{G}$ & $\mathrm{E}$ & $\mathrm{E}$ \\
\hline $\mathbf{E}_{531}$ & $\mathrm{M}$ & $\mathrm{M}$ & $\mathrm{FH}$ & $\mathrm{H}$ & FL & $\mathrm{G}$ & $\mathrm{E}$ & $\mathrm{F}$ & $\mathrm{G}$ & $\mathrm{F}$ \\
\hline $\mathbf{E}_{532}$ & $\mathrm{H}$ & FL & $\mathrm{H}$ & $\mathrm{M}$ & $\mathrm{H}$ & $\mathrm{F}$ & $\mathrm{F}$ & $\mathrm{VG}$ & $\mathrm{F}$ & $\mathrm{G}$ \\
\hline $\mathbf{E}_{533}$ & $\mathrm{M}$ & $\mathrm{M}$ & $\mathrm{M}$ & $\mathrm{M}$ & $\mathrm{M}$ & $\mathrm{F}$ & $\mathrm{F}$ & $\mathrm{F}$ & G & $\mathrm{G}$ \\
\hline $\mathbf{E}_{541}$ & $\mathrm{M}$ & $\mathrm{H}$ & FL & $\mathrm{M}$ & $\mathrm{M}$ & $\mathrm{VG}$ & $\mathrm{E}$ & $\mathrm{F}$ & $\mathrm{G}$ & $\mathrm{G}$ \\
\hline $\mathbf{E}_{542}$ & $\mathrm{H}$ & $\mathrm{H}$ & $\mathrm{FH}$ & $\mathrm{FH}$ & $\mathrm{H}$ & $\mathrm{G}$ & 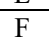 & $\mathrm{F}$ & $\mathrm{F}$ & $\mathrm{VG}$ \\
\hline $\mathbf{E}_{543}$ & $\mathrm{M}$ & $\mathrm{H}$ & $\mathrm{M}$ & $\mathrm{VL}$ & $\mathrm{VL}$ & $\mathrm{G}$ & $\mathrm{F}$ & $\mathrm{F}$ & $\mathrm{F}$ & VP \\
\hline
\end{tabular}

- Step 3: Approximation of the linguistic terms by the corresponding fuzzy intervals [32]: These linguistic variables were approximated by fuzzy intervals [5] chosen from literature $[5,25]$ and presented in Table 4.

Table 4: Linguistic variables and fuzzy numbers for weighting and rating of agility (Adapted from [25])

\begin{tabular}{|c|c|c|c|}
\hline \multicolumn{2}{|c|}{ Importance Weight } & \multicolumn{2}{c|}{ Performance Rating } \\
\hline $\begin{array}{c}\text { Linguistic } \\
\text { variable }\end{array}$ & Fuzzy number & $\begin{array}{c}\text { Linguistic } \\
\text { variable }\end{array}$ & $\begin{array}{c}\text { Fuzzy } \\
\text { number }\end{array}$ \\
\hline Very Low (VL) & $(0,0.05,0.15)$ & Worst (W) & $(0,0.5,1.5)$ \\
\hline Low (L) & $(0.1,0.2,0.3)$ & Very Poor (VP) & $(1,2,3)$ \\
\hline Fairly Low (FL) & $(0.2,0.35,0.5)$ & Poor (P) & $(2,3.5,5)$ \\
\hline Medium (M) & $(0.3,0.5,0.7)$ & Fair (F) & $(3,5,7)$ \\
\hline Fairly High (FH) & $(0.5,0.65,0.8)$ & Good (G) & $(5,6.5,8)$ \\
\hline High (H) & $(0.7,0.8,0.9)$ & Very Good (VG) & $(7,8,9)$ \\
\hline Very High (VH) & $(0.85,0.95,1.0)$ & Excellent (E) & $\begin{array}{c}(8.5,9.5, \\
10\end{array}$ \\
\hline
\end{tabular}


To calculate the average fuzzy weight and performance rating of each attribute [5], the literature recommended using average operation method $[5,27]$.

Example: Average fuzzy weight of the attribute $\mathrm{E}_{111}=$ $[\mathrm{H}+\mathrm{FH}+\mathrm{M}+\mathrm{H}+\mathrm{FH}] / 5=(0.7,0.8,0.9) / 5,(0.5,0.65,0.8) / 5,(0.3,0.5$, $0.7) / 5,(0.7,0.8,0.9) / 5,(0.5,0.65,0.8) / 5=(0.54,0.68,0.82)$

Example: Average fuzzy performance rating of the attribute $\mathrm{E}_{111}=$ $[\mathrm{E}+\mathrm{VG}+\mathrm{VG}+\mathrm{G}+\mathrm{F}] / 5=(8.5,9.5,10) / 5,(7,8,9) / 5,(7,8,9) / 5,(5$, $6.5,8) / 5,(3,5,7) / 5=(6.1,7.4,8.6)$

The following step consists of calculating the rating of each criterion [5]. An example of this calculation for the criterion $E_{11}$ is shown below.

Example: Rating of the criterion

$$
\mathrm{E}_{11}=\frac{\sum_{k=1}^{k=4} \text { (Average fuzzy performance rating } \otimes \text { Average fuzzy weight) }}{\sum_{k=1}^{k=4} \text { Average fuzzy weight }}
$$

$=[(6.1,7.4,8.6) \otimes(0.54,0.68,0.82) \oplus(3.4,5.0,6.6) \otimes(0.54,0.68$, $0.82) \oplus(2.4,3.8,5.3) \otimes(0.58,0.71,0.84) \oplus(4.9,6.5,8.0) \otimes(0.54$, $0.68,0.82)] /[(0.54,0.68,0.82) \oplus(0.54,0.68,0.82) \oplus(0.58,0.71$, $0.84) \oplus(0.54,0.68,0.82)]=(4.17,5.65,7.11)$

By using R language, fuzzy calculations are presented in Table 5.

Table 5: Fuzzy index of agile criteria rating

\begin{tabular}{|c|c|c|c|c|}
\hline 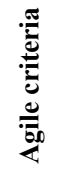 & 递 & $\begin{array}{l}\text { Average fuzzy } \\
\text { performance } \\
\text { rating }\end{array}$ & $\begin{array}{c}\text { Average fuzzy } \\
\text { weight }\end{array}$ & $\begin{array}{l}\text { Criteria } \\
\text { rating }\end{array}$ \\
\hline \multirow{4}{*}{$\mathbf{E}_{11}$} & $E_{111}$ & $(6.1,7.4,8.6)$ & $(0.54,0.68,0.82)$ & \multirow{4}{*}{$\begin{array}{c}(4.17,5.65, \\
7.11)\end{array}$} \\
\hline & $E_{112}$ & $(3.4,5.0,6.6)$ & $(0.54,0.68,0.82)$ & \\
\hline & $E_{113}$ & $(2.4,3.8,5.3)$ & $(0.58,0.71,0.84)$ & \\
\hline & $E_{114}$ & $(4.9,6.5,8.0)$ & $(0.54,0.68,0.82)$ & \\
\hline \multirow{5}{*}{$\mathbf{E}_{12}$} & $E_{121}$ & $(5.0,6.5,8.0)$ & $(0.46,0.62,0.78)$ & \multirow{5}{*}{$\begin{array}{c}(3.52,4.88, \\
6.28)\end{array}$} \\
\hline & $E_{122}$ & $(3.0,4.7,6.4)$ & $(0.54,0.68,0.82)$ & \\
\hline & $E_{123}$ & $(1.4,2.6,4.0)$ & $(0.50,0.65,0.80)$ & \\
\hline & $E_{124}$ & $(1.4,2.6,4.0)$ & $(0.58,0.71,0.84)$ & \\
\hline & $E_{125}$ & $(6.8,8.0,9.0)$ & $(0.58,0.71,0.84)$ & \\
\hline \multirow{7}{*}{$\mathbf{E}_{13}$} & $E_{131}$ & $(5.0,6.5,8.0)$ & $(0.76,0.86,0.94)$ & \multirow{7}{*}{$\begin{array}{c}(4.49,5.99 \\
7.47)\end{array}$} \\
\hline & $E_{132}$ & $(6.7,8.0,9.0)$ & $(0.58,0.71,0.84)$ & \\
\hline & $E_{133}$ & $(5.4,6.8,8.2)$ & $(0.62,0.74,0.86)$ & \\
\hline & $E_{134}$ & $(3.8,5.6,7.4)$ & $(0.42,0.59,0.76)$ & \\
\hline & $E_{135}$ & $(5.0,6.5,8.0)$ & $(0.46,0.62,0.78)$ & \\
\hline & $E_{136}$ & $(2.6,4.1,5.6)$ & $(0.79,0.89,0.96)$ & \\
\hline & $E_{137}$ & $(3.2,4.7,6.3)$ & $(0.54,0.68,0.82)$ & \\
\hline \multirow{5}{*}{$\mathbf{E}_{21}$} & $E_{211}$ & $(4.6,6.2,7.8)$ & $(0.66,0.77,0.88)$ & \multirow{5}{*}{$\begin{array}{c}(3.66,4.93 \\
6.23)\end{array}$} \\
\hline & $E_{212}$ & $(1.8,2.9,4.2)$ & $(0.42,0.59,0.76)$ & \\
\hline & $E_{213}$ & $(2.0,3.5,5.0)$ & $(0.50,0.65,0.80$ & \\
\hline & $E_{214}$ & $(6.4,7.7,8.8)$ & $(0.76,0.86,0.94)$ & \\
\hline & $E_{215}$ & $(1.8,3.2,4.7)$ & $(0.58,0.71,0.84)$ & \\
\hline \multirow{4}{*}{$\mathbf{E}_{22}$} & $E_{221}$ & $(5.3,6.8,8.2)$ & $(0.24,0.41,0.58)$ & \multirow{4}{*}{$\begin{array}{c}(4.45,5.77, \\
7.04)\end{array}$} \\
\hline & $\mathrm{E}_{222}$ & $(3.9,5.0,6.1)$ & $(0.54,0.68,0.82)$ & \\
\hline & $E_{223}$ & $(3.1,4.4,5.7)$ & $(0.50,0.65,0.80)$ & \\
\hline & $E_{224}$ & $(5.7,7.1,8.4)$ & $(0.62,0.74,0.86)$ & \\
\hline \multirow{4}{*}{$\mathbf{E}_{23}$} & $E_{231}$ & $(4.2,5.9,7.6)$ & $(0.76,0.86,0.94)$ & \multirow{4}{*}{$\begin{array}{c}(3.56,5.05, \\
6.55)\end{array}$} \\
\hline & $E_{232}$ & $(2.2,3.5,4.9)$ & $(0.54,0.68,0.82)$ & \\
\hline & $E_{233}$ & $(2.4,3.8,5.3)$ & $(0.7,0.8,0.9)$ & \\
\hline & $E_{234}$ & $(5.7,7.1,8.4)$ & $(0.50,0.65,0.80)$ & \\
\hline $\mathbf{E}_{24}$ & $\mathrm{E}_{241}$ & $(3.4,5.0,6.6)$ & $(0.50,0.65,0.80)$ & $\begin{array}{c}(3.4,5.0 \\
6.6)\end{array}$ \\
\hline \multirow{3}{*}{$\mathbf{E}_{25}$} & $\mathrm{E}_{251}$ & $(5.8,7.1,8.4)$ & $(0.50,0.65,0.80)$ & \multirow{3}{*}{$\begin{array}{c}(3.68,4.89, \\
6.18)\end{array}$} \\
\hline & $\mathrm{E}_{252}$ & $(3.8,5.3,6.8)$ & $(0.50,0.65,0.80)$ & \\
\hline & $E_{253}$ & $(1.0,2.0,3.2)$ & $(0.42,0.59,0.76)$ & \\
\hline $\mathbf{E}_{26}$ & $E_{261}$ & $(5.7,7.1,8.4)$ & $(0.54,0.68,0.82)$ & \\
\hline
\end{tabular}

\begin{tabular}{|c|c|c|c|c|}
\hline & $E_{262}$ & $(2.6,3.8,5.2)$ & $(0.58,0.71,0.84)$ & \multirow{3}{*}{$\begin{array}{c}(3.29,4.63 \\
6.03)\end{array}$} \\
\hline & $\mathrm{E}_{263}$ & $(1.6,2.9,4.3)$ & $(0.50,0.65,0.80)$ & \\
\hline & $E_{264}$ & $(3.2,4.7,6.2)$ & $(0.54,0.68,0.82)$ & \\
\hline \multirow{4}{*}{$\mathbf{E}_{31}$} & $E_{311}$ & $(2.4,3.8,5.3)$ & $(0.58,0.71,0.84)$ & \multirow{4}{*}{$\begin{array}{c}(3.08,4.57 \\
6.06)\end{array}$} \\
\hline & $E_{312}$ & $(1.6,2.9,4.4)$ & $(0.7,0.8,0.9)$ & \\
\hline & $E_{313}$ & $(2.8,4.4,6.0)$ & $(0.79,0.89,0.96)$ & \\
\hline & $E_{314}$ & $(6.4,7.7,8.8)$ & $(0.50,0.65,0.80)$ & \\
\hline \multirow{2}{*}{$\mathbf{E}_{32}$} & $E_{321}$ & $(6.0,7.4,8.6)$ & $(0.7,0.8,0.9)$ & \multirow{2}{*}{$\begin{array}{c}(5.09,6.55, \\
7.92)\end{array}$} \\
\hline & $E_{322}$ & $(4.0,5.6,7.2)$ & $(0.58,0.71,0.84)$ & \\
\hline \multirow{5}{*}{$\mathbf{E}_{33}$} & $E_{331}$ & $(5.7,7.1,8.4)$ & $(0.58,0.71,0.84)$ & \multirow{5}{*}{$\begin{array}{c}(4.69,5.97 \\
7.21)\end{array}$} \\
\hline & $E_{332}$ & $(4.5,6.2,7.8)$ & $(0.7,0.8,0.9)$ & \\
\hline & $\mathrm{E}_{333}$ & $(2.2,3.5,5.0)$ & $(0.38,0.56,0.74)$ & \\
\hline & $E_{334}$ & $(2.2,3.5,4.9)$ & $(0.58,0.71,0.84)$ & \\
\hline & $E_{335}$ & $(7.8,8.9,9.6)$ & $(0.62,0.74,0.86)$ & \\
\hline \multirow{4}{*}{$\mathbf{E}_{34}$} & $E_{341}$ & $(2.8,4.4,6.0)$ & $(0.50,0.65,0.80)$ & \multirow{4}{*}{$\begin{array}{c}(4.21,5.79 \\
7.32)\end{array}$} \\
\hline & $E_{342}$ & $(3.6,5.3,7.0)$ & $(0.50,0.65,0.80)$ & \\
\hline & $E_{343}$ & $(5.3,6.8,8.2)$ & $(0.54,0.68,0.82)$ & \\
\hline & $\mathrm{E}_{344}$ & $(4.9,6.5,8.0)$ & $(0.62,0.74,0.86)$ & \\
\hline \multirow{3}{*}{$\mathbf{E}_{35}$} & $E_{351}$ & $(2.6,4.4,6.2)$ & $(0.58,0.71,0.84)$ & \multirow{3}{*}{$\begin{array}{c}(4.20,5.86 \\
7.45)\end{array}$} \\
\hline & $E_{352}$ & $(4.9,6.5,8.0)$ & $(0.42,0.59,0.76)$ & \\
\hline & $\mathrm{E}_{353}$ & $(5.3,6.8,8.2)$ & $(0.58,0.71,0.84)$ & \\
\hline \multirow{3}{*}{$\mathbf{E}_{36}$} & $E_{361}$ & $(1.0,2.0,3.3)$ & $(0.38,0.56,0.74)$ & \multirow{3}{*}{$\begin{array}{c}(5.01,6.04 \\
7.03)\end{array}$} \\
\hline & $E_{362}$ & $(8.2,9.2,9.8)$ & $(0.54,0.68,0.82)$ & \\
\hline & $E_{363}$ & $(4.7,6.2,7.6)$ & $(0.66,0.77,0.88)$ & \\
\hline \multirow{6}{*}{$\mathbf{E}_{41}$} & $\mathrm{E}_{411}$ & $(6.4,7.7,8.8)$ & $(0.42,0.59,0.76)$ & \multirow{6}{*}{$\begin{array}{c}(3.81,5.30 \\
6.74)\end{array}$} \\
\hline & $\mathrm{E}_{412}$ & $(2.6,4.1,5.7)$ & $(0.46,0.62,0.78)$ & \\
\hline & $\mathrm{E}_{413}$ & $(4.9,6.5,8.0)$ & $(0.42,0.59,0.76)$ & \\
\hline & $\mathrm{E}_{414}$ & $(4.2,5.9,7.6)$ & $(0.66,0.77,0.88)$ & \\
\hline & $\mathrm{E}_{415}$ & $(3.9,5.3,6.6)$ & $(0.58,0.71,0.84)$ & \\
\hline & $\mathrm{E}_{416}$ & $(1.4,2.6,3.9)$ & $(0.54,0.68,0.82)$ & \\
\hline & $\mathrm{E}_{421}$ & $(6.4,7.7,8.8)$ & $(0.73,0.83,0.92)$ & \\
\hline $\mathbf{E}_{42}$ & $\mathrm{E}_{422}$ & $(6.4,7.7,8.8)$ & $(0.50,0.65,0.80)$ & $\begin{array}{l}21,1.52, \\
8677\end{array}$ \\
\hline & $\mathrm{E}_{423}$ & $(5.7,7.1,8.4)$ & $(0.46,0.62,0.78)$ & \\
\hline & $\mathrm{E}_{431}$ & $(6.8,8.0,9.0)$ & $(0.62,0.74,0.86)$ & \\
\hline & $\mathrm{E}_{432}$ & $(7.2,8.3,9.2)$ & $(0.16,0.29,0.43)$ & $(5.84,7.14$, \\
\hline $\mathbf{E}_{43}$ & $\mathrm{E}_{433}$ & $(6.4,7.7,8.8)$ & $(0.44,0.59,0.74)$ & $8.27)$ \\
\hline & $\mathrm{E}_{434}$ & $(4.0,5.3,6.6)$ & $(0.57,0.71,0.84)$ & \\
\hline & $\mathrm{E}_{441}$ & $(6.9,8.0,9.0)$ & $(0.60,0.74,0.86)$ & \\
\hline & $\mathrm{E}_{442}$ & $(4.9,6.2,7.4)$ & $(0.58,0.71,0.84)$ & $(5.14,6.53$, \\
\hline $\mathbf{E}_{44}$ & $\mathrm{E}_{443}$ & $(3.4,5.0,6.6)$ & $(0.59,0.71,0.82)$ & \\
\hline & $\mathrm{E}_{444}$ & $(5.3,6.8,8.2)$ & $(0.72,0.83,0.92)$ & \\
\hline & $\mathrm{E}_{451}$ & $(2.4,3.8,5.3)$ & $(0.72,0.83,0.92)$ & \\
\hline & $\mathrm{E}_{452}$ & $(2.2,3.5,5.0)$ & $(0.79,0.89,0.96)$ & \\
\hline $\mathbf{E}_{45}$ & $\mathrm{E}_{453}$ & $(3.6,5.0,6.4)$ & $(0.60,0.74,0.86)$ & $0.34,4.10$, \\
\hline & $\mathrm{E}_{454}$ & $(2.8,4.4,6.0)$ & $(0.68,0.80,0.90)$ & \\
\hline & $\mathrm{E}_{455}$ & $(6.1,7.4,8.6)$ & $(0.65,0.77,0.88)$ & \\
\hline & $\mathrm{E}_{461}$ & $(3.8,5.6,7.4)$ & $(0.38,0.53,0.68)$ & $(5.28,6.76$, \\
\hline $\mathbf{E}_{46}$ & $\mathrm{E}_{462}$ & $(6.4,7.7,8.8)$ & $(0.50,0.65,0.80)$ & \\
\hline & $\mathrm{E}_{471}$ & $(5.0,6.5,8.0)$ & $(0.66,0.77,0.88)$ & \\
\hline $\mathbf{F}_{17}$ & $\mathrm{E}_{472}$ & $(7.5,8.6,9.4)$ & $(0.36,0.50,0.64)$ & $(5.01,6.42$, \\
\hline $\mathbf{E}_{47}$ & $\mathrm{E}_{473}$ & $(5.3,6.8,8.2)$ & $(0.62,0.74,0.86)$ & 7.74) \\
\hline & $\mathrm{E}_{474}$ & $(3.5,4.7,5.9)$ & $(0.71,0.83,0.92)$ & \\
\hline & $E_{511}$ & $(3.4,5.3,7.2)$ & $(0.34,0.50,0.66)$ & \\
\hline $\mathbf{E}_{51}$ & $\mathrm{E}_{512}$ & $(5.2,6.5,7.6)$ & $(0.54,0.68,0.82)$ & $\begin{array}{c}(0.16,1.34, \\
837)\end{array}$ \\
\hline & $\mathrm{E}_{513}$ & $(8.5,9.5,10.0)$ & $(0.62,0.74,0.86)$ & \\
\hline & $E_{521}$ & $(4.9,6.2,7.4)$ & $(0.69,0.80,0.90)$ & \\
\hline $\mathbf{E}_{52}$ & $\mathrm{E}_{522}$ & $(2.0,3.2,4.5)$ & $(0.71,0.83,0.92)$ & $\begin{array}{c}(4.485 .15 \\
693)\end{array}$ \\
\hline & $\mathrm{E}_{523}$ & $(7.5,8.6,9.4)$ & $(0.49,0.62,0.74)$ & \\
\hline & $E_{531}$ & $(4.9,6.5,8.0)$ & $(0.40,0.56,0.72)$ & \\
\hline $\mathbf{E}_{53}$ & $\mathrm{E}_{532}$ & $(4.2,5.9,7.6)$ & $(0.52,0.65,0.78)$ & $\begin{array}{c}(4.33,0.01, \\
767)\end{array}$ \\
\hline & $\mathrm{E}_{533}$ & $(3.8,5.6,7.4)$ & $(0.3,0.5,0.7)$ & \\
\hline & $E_{541}$ & $(5.7,7.1,8.4)$ & $(0.36,0.53,0.70)$ & \\
\hline $\mathbf{E}_{54}$ & $\mathrm{E}_{542}$ & $(4.2,5.9,7.6)$ & $(0.62,0.74,0.86)$ & $\begin{array}{c}(4.38,0.01, \\
757)\end{array}$ \\
\hline & $\mathrm{E}_{543}$ & $(3.0,4.7,6.4)$ & $(0.26,0.38,0.52)$ & \\
\hline
\end{tabular}

In order to calculate the rating of each enabler, we firstly aggregate the five experts' weights and ratings, by using median operation [25], and then we carry out the same calculation as that of the criteria rating (Table 6). An example of the rating of the enabler $E_{1}$ is shown below. 
Example: Rating of the enabler

$$
\mathrm{E}_{1}=\frac{\sum_{\mathrm{j}=1}^{\mathrm{j}=3} \text { (Criteria rating } \otimes \text { Fuzzy importance weight of the agile criteria) }}{\sum_{\mathrm{j}=1}^{\mathrm{j}=3} \text { Fuzzy importance weight of the agile criteria }}
$$

$=[(4.17,5.65,7.11) \otimes(0.5,0.65,0.8) \oplus(3.52,4.88,6.28) \otimes(0.5$, $0.65,0.8) \oplus(4.49,5.99,7.47) \otimes(0.5,0.65,0.8)] /[(0.5,0.65,0.8)$ $\oplus(0.5,0.65,0.8) \oplus(0.5,0.65,0.8)]=(5.14,6.55,7.86)$

Table 6: Fuzzy index of agile enabler rating

\begin{tabular}{|c|c|c|c|c|c|}
\hline 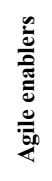 & 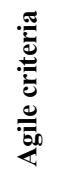 & Criteria rating & $\begin{array}{l}\text { Fuzzy } \\
\text { importance } \\
\text { weight of the } \\
\text { agile criteria }\end{array}$ & $\begin{array}{l}\text { Enabler } \\
\text { rating }\end{array}$ & $\begin{array}{l}\text { Fuzzy } \\
\text { importance } \\
\text { weight of } \\
\text { the agile } \\
\text { enablers }\end{array}$ \\
\hline \multirow{3}{*}{$\mathbf{E}_{1}$} & $E_{11}$ & $(4.17,5.65,7.11)$ & $(0.5,0.65,0.8)$ & \multirow{3}{*}{$\begin{array}{l}(5.14, \\
6.55, \\
7.86)\end{array}$} & \multirow{3}{*}{$\begin{array}{c}(0.5,0.65 \\
0.8)\end{array}$} \\
\hline & $E_{12}$ & $(3.52,4.88,6.28)$ & $(0.5,0.65,0.8)$ & & \\
\hline & $E_{13}$ & $(4.49,5.99,7.47)$ & $(0.5,0.65,0.8)$ & & \\
\hline \multirow{6}{*}{$\mathbf{E}_{2}$} & $E_{21}$ & $(3.66,4.93,6.23)$ & $(0.5,0.65,0.8)$ & \multirow{6}{*}{$\begin{array}{l}(3.67, \\
5.04, \\
6.44)\end{array}$} & \multirow{6}{*}{$\begin{array}{c}(0.5,0.65 \\
0.8)\end{array}$} \\
\hline & $\mathrm{E}_{22}$ & $(4.45,5.77,7.04)$ & $(0.5,0.65,0.8)$ & & \\
\hline & $\mathrm{E}_{23}$ & $(3.56,5.05,6.55)$ & $(0.7,0.8,0.9)$ & & \\
\hline & $\mathrm{E}_{24}$ & $(3.4,5.0,6.6)$ & $(0.5,0.65,0.8)$ & & \\
\hline & $\mathrm{E}_{25}$ & $(3.68,4.89,6.18)$ & $(0.5,0.65,0.8)$ & & \\
\hline & $E_{26}$ & $(3.29,4.63,6.03)$ & $(0.5,0.65,0.8)$ & & \\
\hline \multirow{6}{*}{$\mathbf{E}_{3}$} & $E_{31}$ & $(3.08,4.57,6.06)$ & $(0.7,0.8,0.9)$ & \multirow{6}{*}{$\begin{array}{l}(4.36 \\
5.79 \\
7.16)\end{array}$} & \multirow{6}{*}{$\begin{array}{c}(0.7,0.8 \\
0.9)\end{array}$} \\
\hline & $E_{32}$ & $(5.09,6.55,7.92)$ & $(0.7,0.8,0.9)$ & & \\
\hline & $E_{33}$ & $(4.69,5.97,7.21)$ & $(0.7,0.8,0.9)$ & & \\
\hline & $E_{34}$ & $(4.21,5.79,7.32)$ & $(0.5,0.65,0.8)$ & & \\
\hline & $E_{35}$ & $(4.20,5.86,7.45)$ & $(0.5,0.65,0.8)$ & & \\
\hline & $E_{36}$ & $(5.01,6.04,7.03)$ & $(0.5,0.65,0.8)$ & & \\
\hline \multirow{7}{*}{$\mathbf{E}_{4}$} & $\mathrm{E}_{41}$ & $(3.81,5.30,6.74)$ & $(0.5,0.65,0.8)$ & \multirow{7}{*}{$\begin{array}{l}(4.88, \\
6.31 \\
7.64)\end{array}$} & \multirow{7}{*}{$\begin{array}{c}(0.5,0.65 \\
0.8)\end{array}$} \\
\hline & $\mathrm{E}_{42}$ & $(6.21,7.52,8.67)$ & $(0.5,0.65,0.8)$ & & \\
\hline & $\mathrm{E}_{43}$ & $(5.84,7.14,8.27)$ & $(0.5,0.65,0.8)$ & & \\
\hline & $E_{44}$ & $(5.14,6.53,7.82)$ & $(0.7,0.8,0.9)$ & & \\
\hline & $\mathrm{E}_{45}$ & $(3.34,4.76,6.23)$ & $(0.7,0.8,0.9)$ & & \\
\hline & $\mathrm{E}_{46}$ & $(5.28,6.76,8.16)$ & $(0.5,0.65,0.8)$ & & \\
\hline & $E_{47}$ & $(5.01,6.42,7.74)$ & $(0.7,0.8,0.9)$ & & \\
\hline \multirow{4}{*}{$\mathbf{E}_{5}$} & $E_{51}$ & $(6.16,7.34,8.37)$ & $(0.5,0.65,0.8)$ & \multirow{4}{*}{$\begin{array}{l}(4.90 \\
6.28 \\
7.61)\end{array}$} & \multirow{4}{*}{$\begin{array}{c}(0.5,0.65 \\
0.8)\end{array}$} \\
\hline & $\mathrm{E}_{52}$ & $(4.48,5.75,6.93)$ & $(0.7,0.8,0.9)$ & & \\
\hline & $\mathrm{E}_{53}$ & $(4.33,6.01,7.67)$ & $(0.3,0.5,0.7)$ & & \\
\hline & $E_{54}$ & $(4.38,6.01,7.57)$ & $(0.3,0.5,0.7)$ & & \\
\hline
\end{tabular}

- Step 4: Calculation of the FAI of HealthOrg: We carry out the same calculation as that of the enabler rating [32].

$\mathrm{FAI}=\frac{\sum_{i=1}^{i=5} \text { (Enabler rating } \otimes \text { Fuzzy importance weight of the agile enablers) }}{\sum_{i=1}^{i=5} \text { Fuzzy importance weight of the agile enablers }}$

$=[(5.14,6.55,7.86) \otimes(0.5,0.65,0.8) \oplus(3.67,5.04,6.44) \otimes(0.5$, $0.65,0.8) \oplus(4.36,5.79,7.16) \otimes(0.7,0.8,0.9) \oplus(4.88,6.31,7.64)$ $\otimes(0.5,0.65,0.8) \oplus(4.90,6.28,7.61) \otimes(0.5,0.65,0.8)] /[(0.5$, $0.65,0.8) \oplus(0.5,0.65,0.8) \oplus(0.7,0.8,0.9) \oplus(0.5,0.65,0.8) \oplus$ $(0.5,0.65,0.8)]=(4.57,5.98,7.34)$

The overall agility of HealthOrg is $(4.57,5.98,7.34)$.

- Step 5: Matching the FAI with the appropriate linguistic level [32]: After determining the FAI of the organization, we converted it into linguistic terms. To do this, we used the Euclidean distance method in which we seek to obtain the minimum distance between FAI and the linguistic level (Table 8). Table 7 presents the linguistic terms of different agility levels and their fuzzy intervals [5].
Table 7: Fuzzy values of agility levels (Adapted from [25])

\begin{tabular}{|c|c|}
\hline Level of agility & Fuzzy intervals \\
\hline Slowly Agile & $(0,1.5,3)$ \\
\hline Fairly Agile & $(1.5,3,4.5)$ \\
\hline Agile & $(3.556 .5)$ \\
\hline Very Agile & $(5.5,7,8.5)$ \\
\hline Extremely Agile & $(7,8.5,10)$ \\
\hline
\end{tabular}

Table 8: Agility level of HealthOrg

\begin{tabular}{|l|l|}
\hline FAI for HealthOrg & $(\mathbf{4 . 5 7 , 5 . 9 8 , 7 . 3 4 )}$ \\
\hline D (FAI, Slowly Agile) & $\left\{(4.57-0)^{2}+(5.98-1.5)^{2}+(7.34-3)^{2}\right\}^{1 / 2}=7.78$ \\
\hline D (FAI, Fairly Agile) & $\left\{(4.57-1.5)^{2}+(5.98-3)^{2}+(7.34-4.5)^{2}\right\}^{1 / 2}=5.13$ \\
\hline D (FAI, Agile) & $\left\{(4.57-3.5)^{2}+(5.98-5.0)^{2}+(7.34-6.5)^{2}\right\}^{1 / 2}=1.67$ \\
\hline D (FAI, Very Agile) & $\left\{(4.57-5.5)^{2}+(5.98-7)^{2}+(7.34-8.5)^{2}\right\}^{1 / 2}=1.80$ \\
\hline D (FAI, Extremely Agile) & $\left\{(4.57-7)^{2}+(5.98-8.5)^{2}+(7.34-10)^{2}\right\}^{1 / 2}=4.40$ \\
\hline
\end{tabular}

The minimum distance between FAI and the level of agility is that obtained with the "Agile" level. Then, HealthOrg is considered as an agile enterprise.

- Step 6: Fuzzy performance importance index (FPII) calculation: Although HealthOrg is agile; some attributes weakened its agility during COVID-19 era. In order to identify them, we calculate FPII and the ranking score for each agile attribute (Table 9) [5]. An example of it for E111 is calculated as:

$\mathrm{FPII}_{111}=\left[(1,1,1)-\right.$ Average fuzzy weight of $\left.\mathrm{E}_{111}\right] \otimes$ Average fuzzy performance rating of

$$
\mathrm{E}_{111}=\left[(1,1,1)-\left(\begin{array}{c}
0.540 .680 .82)] \otimes(6.17 .48 .6)=(2.81,2.37, \\
1.55)
\end{array}\right.\right.
$$

\begin{tabular}{|c|c|c|c|c|}
\hline 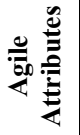 & $\begin{array}{c}\text { Average fuzzy } \\
\text { weight }\end{array}$ & $\begin{array}{c}\text { Fuzzy } \\
\text { performance } \\
\text { average rating }\end{array}$ & FPII & 些 \\
\hline $\mathbf{E}_{111}$ & $(0.54,0.68,0.82)$ & $(6.1,7.4,8.6)$ & $(2.81,2.37,1.55)$ & 2.31 \\
\hline $\mathbf{E}_{112}$ & $(0.54,0.68,0.82)$ & $(3.4,5.0,6.6)$ & $(1.56,1.60,1.19)$ & 1.52 \\
\hline $\mathbf{E}_{113}$ & $(0.58,0.71,0.84)$ & $(2.4,3.8,5.3)$ & $(1.011 .10,0.85)$ & 1.04 \\
\hline $\mathbf{E}_{114}$ & $(0.54,0.68,0.82)$ & $(4.9,6.5,8.0)$ & $(2.25,2.08,1.44)$ & 2.00 \\
\hline$E_{121}$ & $(0.46,0.62,0.78)$ & $(5.0,6.5,8.0)$ & $(2.702 .471 .76)$ & 2.39 \\
\hline $\mathbf{E}_{122}$ & $(0.54,0.68,0.82)$ & $(3.0,4.7,6.4)$ & $(1.38,1.50,1.15)$ & 1.42 \\
\hline$E_{123}$ & $(0.50,0.65,0.80)$ & $(1.4,2.6,4.0)$ & $(0.70,0.91,0.80)$ & 0.86 \\
\hline$E_{124}$ & $(0.58,0.71,0.84)$ & $(1.4,2.6,4.0)$ & $(0.59,0.75,0.64)$ & 0.70 \\
\hline $\mathbf{E}_{125}$ & $(0.58,0.71,0.84)$ & $(6.8,8.0,9.0)$ & $(2.86,2.32,1.44)$ & 2.26 \\
\hline $\mathbf{E}_{131}$ & $(0.76,0.86,0.94)$ & $(5.0,6.5,8.0)$ & $(1.20,0.91,0.48)$ & 0.89 \\
\hline$E_{132}$ & $(0.58,0.71,0.84)$ & $(6.7,8.0,9.0)$ & $(2.81,2.32,1.44)$ & 2.25 \\
\hline $\mathbf{E}_{133}$ & $(0.62,0.74,0.86)$ & $(5.4,6.8,8.2)$ & $(2.05,1.77,1.15)$ & 1.71 \\
\hline $\mathbf{E}_{134}$ & $(0.42,0.59,0.76)$ & $(3.8,5.6,7.4)$ & $(2.20,2.30,1.78)$ & 2.20 \\
\hline$E_{135}$ & $(0.46,0.62,0.78)$ & $(5.0,6.5,8.0)$ & $(2.70,2.47,1.76)$ & 2.39 \\
\hline $\mathbf{E}_{136}$ & $(0.79,0.89,0.96)$ & $(2.6,4.1,5.6)$ & $(0.55,0.45,0.22)$ & 0.43 \\
\hline $\mathbf{E}_{137}$ & $(0.54,0.68,0.82)$ & $(3.2,4.7,6.3)$ & $(1.47,1.50,1.13)$ & 1.43 \\
\hline $\mathbf{E}_{211}$ & $(0.66,0.77,0.88)$ & $(4.6,6.2,7.8)$ & $(1.56,1.43,0.94)$ & 1.37 \\
\hline $\mathbf{E}_{212}$ & $(0.42,0.59,0.76)$ & $(1.8,2.9,4.2)$ & $(1.04,1.19,1.01)$ & 1.13 \\
\hline$E_{213}$ & $(0.50,0.65,0.80$ & $(2.0,3.5,5.0)$ & $(1.00,1.22,1.00)$ & 1.15 \\
\hline$E_{214}$ & $(0.76,0.86,0.94)$ & $(6.4,7.7,8.8)$ & $(1.54,1.08,0.53)$ & 1.06 \\
\hline$E_{215}$ & $(0.58,0.71,0.84)$ & $(1.8,3.2,4.7)$ & $(0.76,0.93,0.75)$ & 0.87 \\
\hline$E_{221}$ & $(0.24,0.41,0.58)$ & $(5.3,6.8,8.2)$ & $(4.03,4.01,3.44)$ & 3.92 \\
\hline $\mathbf{E}_{222}$ & $(0.54,0.68,0.82)$ & $(3.9,5.0,6.1)$ & $(1.79,1.60,1.10)$ & 1.55 \\
\hline
\end{tabular}

Ranking score of $\mathrm{E}_{111}=(2.81+4 \times 2.37+1.55) / 6=2.31$

Table 9: FPII and ranking score of agile attributes 
F. Tamtam et al. / Advances in Science, Technology and Engineering Systems Journal Vol. 5, No. 4, 567-576 (2020)

\begin{tabular}{|c|c|c|c|c|}
\hline $\mathbf{E}_{223}$ & $(0.50,0.65,0.80)$ & $(3.1,4.4,5.7)$ & $(1.55,1.54,1.14)$ & 1.47 \\
\hline $\mathbf{E}_{224}$ & $(0.62,0.74,0.86)$ & $(5.7,7.1,8.4)$ & $(2.17,1.85,1.18)$ & 1.79 \\
\hline $\mathbf{E}_{231}$ & $(0.76,0.86,0.94)$ & $(4.2,5.9,7.6)$ & $(1.01,0.83,0.46)$ & 0.80 \\
\hline $\mathbf{E}_{232}$ & $(0.54,0.68,0.82)$ & $(2.2,3.5,4.9)$ & $(1.01,1.12,0.88)$ & 1.06 \\
\hline $\mathbf{E}_{233}$ & $(0.7,0.8,0.9)$ & $(2.4,3.8,5.3)$ & $(0.72,0.76,0.53)$ & 0.71 \\
\hline $\mathbf{E}_{234}$ & $(0.50,0.65,0.80)$ & $(5.7,7.1,8.4)$ & $(2.85,2.48,1.68)$ & 2.41 \\
\hline $\mathbf{E}_{241}$ & $(0.50,0.65,0.80)$ & $(3.4,5.0,6.6)$ & $(1.70,1.75,1.32)$ & 1.67 \\
\hline $\mathbf{E}_{251}$ & $(0.50,0.65,0.80)$ & $(5.8,7.1,8.4)$ & $(2.90,2.48,1.68)$ & 2.42 \\
\hline $\mathbf{E}_{252}$ & $(0.50,0.65,0.80)$ & $(3.8,5.3,6.8)$ & $(1.90,1.85,1.36)$ & 1.78 \\
\hline$E_{253}$ & $(0.42,0.59,0.76)$ & $(1.0,2.0,3.2)$ & $\begin{array}{c}(0.58,0.82,0.77) \\
\end{array}$ & 0.77 \\
\hline$E_{261}$ & $(0.54,0.68,0.82)$ & $(5.7,7.1,8.4)$ & $(2.62,2.27,1.51)$ & 2.20 \\
\hline$E_{262}$ & $(0.58,0.71,0.84)$ & $(2.6,3.8,5.2)$ & $(1.09,1.10,0.83)$ & 1.05 \\
\hline$E_{263}$ & $(0.50,0.65,0.80)$ & $(1.6,2.9,4.3)$ & $(0.80,1.01,0.86)$ & 0.95 \\
\hline $\mathbf{E}_{264}$ & $(0.54,0.68,0.82)$ & $(3.2,4.7,6.2)$ & $(1.47,1.50,1.12)$ & 1.43 \\
\hline $\mathbf{E}_{311}$ & $(0.58,0.71,0.84)$ & $(2.4,3.8,5.3)$ & $(1.01,1.10,0.85)$ & 1.04 \\
\hline $\mathbf{E}_{312}$ & $(0.7,0.8,0.9)$ & $(1.6,2.9,4.4)$ & $(0.48,0.58,0.44)$ & 0.54 \\
\hline $\mathbf{E}_{313}$ & $\begin{array}{l}(0.79,0.89,0.96) \\
\end{array}$ & $(2.8,4.4,6.0)$ & $(0.59,0.48,0.24)$ & 0.46 \\
\hline $\mathbf{E}_{314}$ & $(0.50,0.65,0.80)$ & $(6.4,7.7,8.8)$ & $(3.20,2.69,1.76)$ & 2.62 \\
\hline$E_{321}$ & $(0.7,0.8,0.9)$ & $(6.0,7.4,8.6)$ & $(1.80,1.48,0.86)$ & 1.43 \\
\hline $\mathbf{E}_{322}$ & $(0.58,0.71,0.84)$ & $(4.0,5.6,7.2)$ & $(1.68,1.62,1.15)$ & 1.55 \\
\hline $\mathbf{E}_{331}$ & $(0.58,0.71,0.84)$ & $(5.7,7.1,8.4)$ & $(2.39,2.06,1.34)$ & 1.99 \\
\hline $\mathbf{E}_{332}$ & $(0.7,0.8,0.9)$ & $(4.5,6.2,7.8)$ & $(1.35,1.24,0.78)$ & 1.18 \\
\hline $\mathbf{E}_{333}$ & $(0.38,0.56,0.74)$ & $(2.2,3.5,5.0)$ & $(1.36,1.54,1.30)$ & 1.47 \\
\hline $\mathbf{E}_{334}$ & $(0.58,0.71,0.84)$ & $(2.2,3.5,4.9)$ & $(0.92,1.01,0.78)$ & 0.96 \\
\hline$E_{335}$ & $(0.62,0.74,0.86)$ & $(7.8,8.9,9.6)$ & $(2.96,2.31,1.34)$ & 2.26 \\
\hline $\mathbf{E}_{341}$ & $(0.50,0.65,0.80)$ & $(2.8,4.4,6.0)$ & $(1.40,1.54,1.20)$ & 1.46 \\
\hline$E_{342}$ & $(0.50,0.65,0.80)$ & $(3.6,5.3,7.0)$ & $(1.80,1.85,1.40)$ & 1.77 \\
\hline $\mathbf{E}_{343}$ & $(0.54,0.68,0.82)$ & $(5.3,6.8,8.2)$ & $(2.44,2.18,1.48$ & 2.11 \\
\hline $\mathbf{E}_{344}$ & $(0.62,0.74,0.86)$ & $(4.9,6.5,8.0)$ & $(1.86,1.69,1.12)$ & 1.62 \\
\hline$E_{351}$ & $(0.58,0.71,0.84)$ & $(2.6,4.4,6.2)$ & $(1.09,1.28,0.99)$ & 1.20 \\
\hline $\mathbf{E}_{352}$ & $(0.42,0.59,0.76)$ & $(4.9,6.5,8.0)$ & $(2.84,2.66,1.92)$ & 2.57 \\
\hline $\mathbf{E}_{353}$ & $(0.58,0.71,0.84)$ & $(5.3,6.8,8.2)$ & $(2.23,1.97,1.31)$ & 1.90 \\
\hline $\mathbf{E}_{361}$ & $(0.38,0.56,0.74)$ & $(1.0,2.0,3.3)$ & $(0.62,0.88,0.86)$ & 0.83 \\
\hline $\mathbf{E}_{362}$ & $(0.54,0.68,0.82)$ & $(8.2,9.2,9.8)$ & $(3.77,2.94,1.76)$ & 2.88 \\
\hline $\mathbf{E}_{363}$ & $(0.66,0.77,0.88)$ & $(4.7,6.2,7.6)$ & $(1.60,1.43,0.91)$ & 1.37 \\
\hline $\mathbf{E}_{411}$ & $(0.42,0.59,0.76)$ & $(6.4,7.7,8.8)$ & $(3.71,3.16,2.11)$ & 3.08 \\
\hline $\mathbf{E}_{412}$ & $(0.46,0.62,0.78)$ & $(2.6,4.1,5.7)$ & $(1.40,1.56,1.25)$ & 1.48 \\
\hline $\mathbf{E}_{413}$ & $(0.42,0.59,0.76)$ & $(4.9,6.5,8.0)$ & $(2.84,2.66,1.92)$ & 2.57 \\
\hline $\mathbf{E}_{414}$ & $(0.66,0.77,0.88)$ & $(4.2,5.9,7.6)$ & $(1.43,1.36,0.91)$ & 1.30 \\
\hline $\mathbf{E}_{415}$ & $(0.58,0.71,0.84)$ & $(3.9,5.3,6.6)$ & $(1.64,1.54,1.06)$ & 1.48 \\
\hline $\mathbf{E}_{416}$ & $(0.54,0.68,0.82)$ & $(1.4,2.6,3.9)$ & $(0.64,0.83,0.70)$ & 0.78 \\
\hline $\mathbf{E}_{421}$ & $(0.73,0.83,0.92)$ & $(6.4,7.7,8.8)$ & $(1.73,1.31,0.70)$ & 1.28 \\
\hline $\mathbf{E}_{422}$ & $(0.50,0.65,0.80)$ & $(6.4,7.7,8.8)$ & $(3.20,2.69,1.76)$ & 2.62 \\
\hline $\mathbf{E}_{423}$ & $(0.46,0.62,0.78)$ & $(5.7,7.1,8.4)$ & $(3.08,2.70,1.85)$ & 2.62 \\
\hline $\mathbf{E}_{431}$ & $(0.62,0.74,0.86)$ & $(6.8,8.0,9.0)$ & $(2.58,2.08,1.26)$ & 2.03 \\
\hline $\mathbf{E}_{432}$ & $(0.16,0.29,0.43)$ & $(7.2,8.3,9.2)$ & $(6.05,5.89,5.24)$ & 5.81 \\
\hline $\mathbf{E}_{433}$ & $(0.44,0.59,0.74)$ & $(6.4,7.7,8.8)$ & $(3.58,3.16,2.29)$ & 3.08 \\
\hline $\mathbf{E}_{434}$ & $(0.57,0.71,0.84)$ & $(4.0,5.3,6.6)$ & $(1.72,1.54,1.06)$ & 1.49 \\
\hline $\mathbf{E}_{441}$ & $(0.60,0.74,0.86)$ & $(6.9,8.0,9.0)$ & $(2.76,2.08,1.26)$ & 2.06 \\
\hline $\mathbf{E}_{442}$ & $(0.58,0.71,0.84)$ & $(4.9,6.2,7.4)$ & $(2.06,1.80,1.18)$ & 1.74 \\
\hline $\mathbf{E}_{443}$ & $(0.59,0.71,0.82)$ & $(3.4,5.0,6.6)$ & $(1.39,1.45,1.19)$ & 1.40 \\
\hline $\mathbf{E}_{444}$ & $(0.72,0.83,0.92)$ & $(5.3,6.8,8.2)$ & $(1.48,1.16,0.66)$ & 1.13 \\
\hline $\mathbf{E}_{451}$ & $(0.72,0.83,0.92)$ & $(2.4,3.8,5.3)$ & $(0.67,0.65,0.42)$ & 0.61 \\
\hline $\mathbf{E}_{452}$ & $(0.79,0.89,0.96)$ & $(2.2,3.5,5.0)$ & $(0.46,0.38,0.20)$ & 0.36 \\
\hline $\mathbf{E}_{453}$ & $(0.60,0.74,0.86)$ & $(3.6,5.0,6.4)$ & $(1.44,1.30,0.90)$ & 1.26 \\
\hline $\mathbf{E}_{454}$ & $(0.68,0.80,0.90)$ & $(2.8,4.4,6.0)$ & $(0.90,0.88,0.60)$ & 0.84 \\
\hline $\mathbf{E}_{455}$ & $(0.65,0.77,0.88)$ & $(6.1,7.4,8.6)$ & $(2.13,1.70,1.03)$ & 1.66 \\
\hline $\mathbf{E}_{461}$ & $(0.38,0.53,0.68)$ & $(3.8,5.6,7.4)$ & $(2.36,2.63,2.37)$ & 2.54 \\
\hline $\mathbf{E}_{462}$ & $(0.50,0.65,0.80)$ & $(6.4,7.7,8.8)$ & $(3.20,2.69,1.76)$ & 2.62 \\
\hline $\mathbf{E}_{471}$ & $(0.66,0.77,0.88)$ & $(5.0,6.5,8.0)$ & $(1.70,1.49,0.96)$ & 1.44 \\
\hline $\mathbf{E}_{472}$ & $(0.36,0.50,0.64)$ & $(7.5,8.6,9.4)$ & $(4.80,4.30,3.38)$ & 4.23 \\
\hline $\mathbf{E}_{473}$ & $(0.62,0.74,0.86)$ & $(5.3,6.8,8.2)$ & $(2.01,1.77,1.15)$ & 1.71 \\
\hline $\mathbf{E}_{474}$ & $(0.71,0.83,0.92)$ & $(3.5,4.7,5.9)$ & $(1.01,0.80,0.47)$ & 0.78 \\
\hline $\mathbf{E}_{511}$ & $(0.34,0.50,0.66)$ & $(3.4,5.3,7.2)$ & $(2.24,2.65,2.45)$ & 2.55 \\
\hline $\mathbf{E}_{512}$ & $(0.54,0.68,0.82)$ & $(5.2,6.5,7.6)$ & $(2.39,2.08,1.37)$ & 2.01 \\
\hline $\mathbf{E}_{513}$ & $(0.62,0.74,0.86)$ & $(8.5,9.5,10.0)$ & $(3.23,2.47,1.40)$ & 2.42 \\
\hline $\mathbf{E}_{521}$ & $(0.69,0.80,0.90)$ & $(4.9,6.2,7.4)$ & $(1.52,1.24,0.74)$ & 1.20 \\
\hline$E_{522}$ & $(0.71,0.83,0.92)$ & $(2.0,3.2,4.5)$ & $(0.58,0.54,0.36)$ & 0.52 \\
\hline $\mathbf{E}_{523}$ & $(0.49,0.62,0.74)$ & $(7.5,8.6,9.4)$ & $(3.82,3.27,2.44)$ & 3.22 \\
\hline $\mathbf{E}_{531}$ & $(0.40,0.56,0.72)$ & $(4.9,6.5,8.0)$ & $(2.94,2.86,2.24)$ & 2.77 \\
\hline $\mathbf{E}_{532}$ & $(0.52,0.65,0.78)$ & $(4.2,5.9,7.6)$ & $(2.02,2.06,1.67)$ & 1.99 \\
\hline
\end{tabular}

\begin{tabular}{|c|c|c|c|c|}
\hline $\mathbf{E}_{533}$ & $(0.3,0.5,0.7)$ & $(3.8,5.6,7.4)$ & $(2.66,2.80,2.22)$ & 2.68 \\
\hline $\mathbf{E}_{541}$ & $(0.36,0.53,0.70)$ & $(5.7,7.1,8.4)$ & $(3.65,3.34,2.52)$ & 3.25 \\
\hline $\mathbf{E}_{542}$ & $(0.62,0.74,0.86)$ & $(4.2,5.9,7.6)$ & $(1.60,1.53,1.06)$ & 1.46 \\
\hline $\mathbf{E}_{543}$ & $(0.26,0.38,0.52)$ & $(3.0,4.7,6.4)$ & $(2.22,2.91,3.07)$ & 2.82 \\
\hline
\end{tabular}

Based on the five experts' experience, scale 1.1 was considered as the threshold which distinguishes the weaker attributes than the other ones. Table 10 showed these attributes and some suggestions to improve them [5].

Table 10: Weaker agile attributes and improvement proposals

\begin{tabular}{|c|c|c|}
\hline Weak agile attribute & References & $\begin{array}{c}\text { Improvement } \\
\text { proposals }\end{array}$ \\
\hline $\begin{array}{ll}\text { - } & \text { Staff interchangeability } \\
\text { - } & \text { Multi-skilled and flexible } \\
\text { staff } & \\
\text { - } & \begin{array}{l}\text { Implementation of job } \\
\text { rotation system }\end{array}\end{array}$ & {$[15,33]$} & $\begin{array}{l}\text { Prepare employees } \\
\text { to participate in } \\
\text { the } \\
\text { implementation of } \\
\text { job rotation system }\end{array}$ \\
\hline $\begin{array}{l}\text { - Flexible employees to accept } \\
\text { the adoption of new } \\
\text { technologies } \\
\text { Multi-functional, developed } \\
\text { and trained employees }\end{array}$ & {$[15,29]$} & $\begin{array}{l}\text { Develop a flexible } \\
\text { working } \\
\text { environment for } \\
\text { employees }\end{array}$ \\
\hline \begin{tabular}{llr} 
- & \multicolumn{2}{c}{ Decentralized decision- } \\
making, knowledge and \\
control \\
- $\begin{array}{l}\text { Knowledge and skills } \\
\text { management systems }\end{array}$ \\
- $\begin{array}{l}\text { Staff empowerment to } \\
\text { resolve patient issues }\end{array}$
\end{tabular} & $\begin{array}{c}{[15,29,30,} \\
31,33]\end{array}$ & $\begin{array}{l}\text { Give authority to } \\
\text { different level } \\
\text { employees which } \\
\text { contributes to } \\
\text { improved their } \\
\text { knowledge }\end{array}$ \\
\hline $\begin{array}{ll}\text { - } & \text { Loyalty and commitment to a } \\
\text { project or a group } \\
\text { - } \\
\text { Participative management } \\
\text { style } \\
\text { Quick evaluation and } \\
\text { implementation of employee } \\
\text { suggestions } \\
\text { Involvement of suppliers and } \\
\text { different agents in } \\
\text { product/service development }\end{array}$ & {$[15,29,30]$} & $\begin{array}{l}\text { Remove barriers to } \\
\text { facilitate the } \\
\text { participation of } \\
\text { different } \\
\text { employees and } \\
\text { suppliers }\end{array}$ \\
\hline $\begin{array}{ll}\text { - } & \text { Efficient information system } \\
\text { and technology } \\
\text { - } \\
\text { Exploitation of information } \\
\text { technology (IT) in supply } \\
\text { chain management }\end{array}$ & $\begin{array}{l}{[15,27,29,} \\
30,32,33]\end{array}$ & $\begin{array}{l}\text { Link information } \\
\text { systems to } \\
\text { technology }\end{array}$ \\
\hline 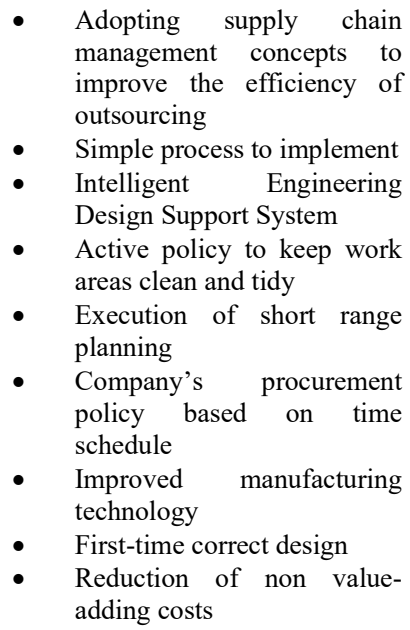 & & $\begin{array}{l}\text { Use advanced } \\
\text { technologies and } \\
\text { production } \\
\text { methods }\end{array}$ \\
\hline
\end{tabular}




\section{Conclusion}

This study evaluated organization agility of a public hospital in Morocco in times of COVID-19. The enablers influencing agility were studied, as were the agile criteria and attributes. After a literature review, an assessment model was presented and tested via the fuzzy logic approach. Empirical results showed that HealthOrg is agile. The COVID-19 outbreak has revealed how different enablers can influenced the hospital agility. It has also shown how some agile enablers need to be enhanced in order to increase the healthcare organization agility.

This article offers initial empirical exploration on how Moroccan healthcare organizations cope with the COVID-19 crisis. It allows identifying the required changes to improve the agility of the organization. There will be increasing improvement for hospitals in technology and human resources departments; COVID-19 has demonstrated their importance in making the healthcare organization extremely agile.

Despite the above benefits for using the assessment model, there is some limitation: this model does not take into account the different agile drivers and capacities which must be aligned with the agile enablers. Also, the organizational agility assessment has been done for a single healthcare organization; however future research should replicate the assessment model in others organizations, in public and private sector. Also, it is highly recommended to compare the results obtained in times of COVID19 with those provided by previous studies. Moreover, further practical suggestions for healthcare sector through COVID 19 outbreaks should be provided.

\section{Conflict of Interest}

The authors declare no conflict of interest.

\section{Acknowledgment}

The authors acknowledge the financial support of the National Centre for Scientific and Technical Research (CNRST) under the Excellence Research Scholarships Program.

\section{References}

[1] K. C. Ng Kee Kwong, P. R. Mehta, G. Shukla, A. R. Mehta, "COVID-19, SARS and MERS: a neurological perspective" Journal of Clinical Neuroscience, 2020. https://doi.org/10.1016/j.jocn.2020.04.124

[2] I. Chakraborty, P. Maity, "COVID-19 outbreak: Migration, effects on society, global environment and prevention" Science of the Total Environment, 728, 2020. https://doi.org/10.1016/j.scitotenv.2020.138882

[3] K. Barro, A. Malone, A. Mokede, C. Chevance, "Management of the COVID-19 epidemic by public health establishments-analysis by the fédération hospitalière de France" Journal of Visceral Surgery, 2020. https://doi.org/10.1016/j.jviscsurg.2020.04.011

[4] Y. Y. Yusuf, M. Sarhadi, A. Gunasekaran, "Agile manufacturing: the drivers, concepts and attributes" International Journal of Production Economics, 62(1-2), 33-43, 1999. https://doi.org/10.1016/S09255273(98)00219-9

[5] M. Suresh, R. Patri, "Agility assessment using fuzzy logic approach: a case of healthcare dispensary" BMC Health Services Reserach, 17(1), 2017. https://doi.org/10.1186/s12913-017-2332-y

[6] M. Christopher, "The agile supply chain: competing in volatile markets" Industrial Marketing Management, 29(1), 37-44, 2000. https://doi.org/10.1016/S0019-8501(99)00110-8

[7] R. Kitzmiller, E. Hunt, S. B. Sproat, "Adopting best practices: 'agility' moves from software development to healthcare project management" Computers Informatics Nursing, 24(2), 75-82, 2006. https://doi.org/10.1097/00024665-200603000-00005

[8] S. Brown, J. Bessant, "The manufacturing strategy-capabilities links in mass customisation and agile manufacturing - an exploratory study" International
Journal of Operations \& Production Management, 23(7), 707-730, 2003, https://doi.org/10.1108/01443570310481522

[9] H. Sharifi, Z. Zhang, "Agile manufacturing in practice - application of a methodology" International Journal of Operations \& Production $\begin{array}{llll}\text { Management, } & \text { 21(5/6), } & 772-794, & 2001 .\end{array}$ https://doi.org/10.1108/01443570110390462

[10] G. Fliedner, R. J. Vokurka, "Agility: competitive weapon of the 1990s and beyond?" Production and inventory management journal, 38(3), 19-24, 1997.

[11] E. Bottani, “A fuzzy QFD approach to achieve agility” International Journal of Production Economics, 119(2), 380-391, 2009. https://doi.org/10.1016/j.ijpe.2009.02.013

[12] J. Prince, J. M. Kay, "Combining lean and agile characteristics: creation of virtual groups by enhanced production flow analysis" International Journal of Production Economics, 85(3), 305-318, 2003. https://doi.org/10.1016/S0925-5273(03)00118-X

[13] P. g. Saleeshya, A. S. Babu, A. s. Vishnu, "A model to assess the agility of manufacturing organisations: systems approach and application" International Journal of Productivity and Quality Management, 8(3), 265295, 2011. https://doi.org/10.1504/IJPQM.2011.042509.

[14] H. Dong, C. L. Li, "Agile supply chain performance evaluation of automobile manufacturing based on regression analysis" Applied Mechanics and Materials, 2662-2666,

2013 https://doi.org/10.4028/www.scientific.net/AMM.397-400.2662

[15] S. Vinodh, S. R. Devadasan, B. Vasudeva Reddy, K. Ravichand, "Agility index measurement using multi-grade fuzzy approach integrated in a 20 criteria agile model" International Journal of Production Research, 48(23), 7159-7176, 2010. https://doi.org/10.1080/00207540903354419

[16] P. G. Saleeshya, A. S. Babu, "A combined AHP- and DEA-based approach to measure agility of manufacturing systems" International Journal of Business and Systems Research, 6(4), 431-455, 2012. https://doi.org/10.1504/IJBSR.2012.049472

[17] S. G. Azevedo, H. Carvalho, V. C. Machado, "Agile index: automotive supply chain" International Journal of Industrial and Manufacturing Engineering, 5(7), 1433-1439, 2011. https://doi.org doi.org/10.5281/zenodo. 1078048

[18] S. A. Raj, A. Sudheer, S. Vinodh, G. Anand, "A mathematical model to evaluate the role of agility enablers and criteria in a manufacturing environment" International Journal of Production Research, 51(19), 59715984, 2013. https://doi.org/10.1080/00207543.2013.825381

[19] K. Khalili-Damghani, M. T. Taghavifard, "A three-stage fuzzy DEA approach to measure performance of a serial process including JIT practices, agility indices, and goals in supply chains" International Journal of Services and Operations Management, 13(2), 147-188, 2012. https://doi.org/10.1504/ijsom.2012.048828

[20] R. Zitkiene, M. Deksnys, "Organizational agility conceptual model" Montenegrin Journal of Economics, 14(2), 115-129, 2018 https://doi.org/10.14254/1800-5845/2018.14-2.7

[21] A. Gunasekaran, "Agile manufacturing: enablers and an implementation framework" International Journal of Production Research, 36(5), 1223 1247, 1998. https://doi.org/10.1080/002075498193291

[22] M. Nejatian, M. H. Zarei, "Moving towards organizational agility: are we improving in the right direction?" Global Journal of Flexible Systems Management, 14(4), 241-253, 2013. https://doi.org/10.1007/s40171-0130048-3

[23] Z. Zhang, H. Sharifi, “A methodology for achieving agility in manufacturing organisations" International Journal of Operations \& Production Management, 20(4), 496-513, 2000 https://doi.org/10.1108/01443570010314818

[24] S. Tolf, M. E. Nyström, C. Tishelman, M. Brommels, J. Hansson, “Agile, a guiding principle for health care improvement?" International Journal of Health Care Quality Assurance, 28(5), 468-493, 2015. https://doi.org/10.1108/IJHCQA-04-2014-0044

[25] C.-T. Lin, H. Chiu, Y.-H. Tseng, "Agility evaluation using fuzzy logic" International Journal of Production Economics, 101(2), 353-368, 2006. https://doi.org/10.1016/j.ijpe.2005.01.011.

[26] W. Triaa, L. Gzara, H. Verjus, "Organizational agility key factors for dynamic business process management" in 2016 IEEE 18th Conference on Business Informatics (CBI), Paris, France, 2016 https://doi.org/10.1109/CBI.2016.16

[27] A. T. Eshlaghy, A. N. Mashayekhi, A. Rajabzadeh, M. M. Razavian, "Applying path analysis method in defining effective factors in organisation agility" International Journal of Production Research, 48(6), 1765-1786, 2010. https://doi.org/10.1080/00207540802566410

[28] C.-S. Tsai, C.-W. Chen, C.-T. Li, "Align agile drivers, capabilities and providers to achieve agility: a fuzzy-logic QFD approach" Supply Chain, 2008.

[29] D. Vázquez-Bustelo, L. Avella, E. Fernández, "Agility drivers, enablers and outcomes: empirical test of an integrated agile manufacturing model" 
International journal of operations \& production management, 27(12), 13031332, 2007. https://doi.org/10.1108/01443570710835633

[30] B. Sherehiy, W. Karwowski, J. K. Layer, "A review of enterprise agility: concepts, frameworks, and attributes," International Journal of Industrial Ergonomics, 37(5), $345-460, \quad 2007$. https://doi.org/10.1016/j.ergon.2007.01.007

[31] R. Patri, M. Suresh, "Modelling the Enablers of Agile Performance in Healthcare Organization: A TISM Approach" Global Journal of Flexible Systems Management, 18(3), 251-272, 2017. https://doi.org/10.1007/s40171-017-0160-x

[32] M. Deksnys, "Organizational agility in high growth companies" $\mathrm{Ph} . \mathrm{D}$ Thesis, Mykolas Romeris University, 2018.

[33] Yuh-Chuan Shih, Ching-Torng Lin, "Agility index of manufacturing firm-a fuzzy-logic-based approach" in 2002 IEEE International Engineering Management Conference, Cambridge, UK, 2002. https://doi.org/10.1109/IEMC.2002.1038476

[34] S. Vinodh, S. R. Devadasan, "Twenty criteria based agility assessment using fuzzy logic approach" International Journal of Advanced Manufacturing Technology, 54(9), 1219-1231, 2011. https://doi.org/10.1007/s00170-0103015-6

[35] V. Vaishnavi, M. Suresh, "Assessing the readiness level of healthcare for implementing agility using fuzzy logic approach," Global Journal of Flexible Systems Management, 21(2), 163-189, 2020. https://doi.org/10.1007/s40171-020-00237-7 